\title{
Utilizing network pharmacology and molecular docking to explore the underlying mechanism of Guizhi Fuling Wan in treating endometriosis
}

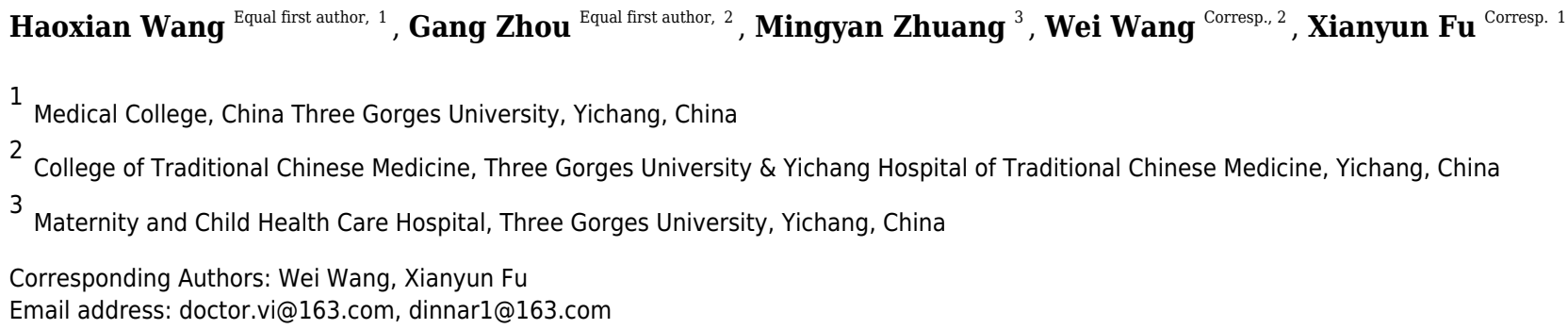

Background. Guizhi Fuling Wan (GZFLW) is a widely used classical Chinese herbal formulae prescribed for the treatment of endometriosis (EMs). This study aimed to predict the key targets and mechanisms of GZFLW in the treatment of EMs by network pharmacology and molecular docking. Methods. Firstly, related compounds and targets of GZFLW were identified through the TCMSP, BATMAN-TCM and CASC Database. Then, the EMs target database was built by GeneCards. The overlapping targets between GZFLW and EMs were screened out, and then data of the PPI network was obtained by the String database to analyze the interrelationship of these targets. Furthermore, a topological analysis was performed to screen the hub targets. After that, molecular docking technology was used to confirm the binding degree of the main active compounds and hub targets. Finally, the DAVID database and Metascape database were used for GO and KEGG enrichment analysis. Results. A total of 89 GZFLW compounds and 284 targets were collected. One hundred one matching targets were picked out as the correlative targets of GZFLW in treating EMs. Among these, 25 significant hub targets were recognized by the PPI network. Coincidently, molecular docking simulation indicated that the hub targets had a good bonding activity with most active compounds (69.71\%). Furthermore, 116 items, including the inflammatory reaction, RNA polymerase, DNA transcription, growth factor activity, and steroid-binding, were selected by GO enrichment analysis. Moreover, the KEGG enrichment analysis results included 100 pathways focused on the AGE-RAGE pathway, HIF pathway, PI3K Akt pathway, MAPK pathway, and TP53 pathway, which exposed the potential mechanisms of GZFLW in treating EMs. Conclusion. Through the exploration of network pharmacology and molecular docking technology, GZFLW has a therapeutic effect on EMs through multi-target mechanism. This study provided a good foundation for further experimental research.

Peer) reviewing PDF | (2020:06:49732:2:0:NEW 9 Feb 2021) 


\section{Utilizing network pharmacology and molecular}

\section{2 docking to explore the underlying mechanism of}

3 Guizhi Fuling Wan in treating endometriosis

4

5

6 Haoxian Wang ${ }^{1}$, Gang Zhou ${ }^{2}$, Mingyan Zhuang ${ }^{3}$, Wei Wang ${ }^{2}$, Xianyun Fu ${ }^{1}$

7

$8{ }^{1}$ Medical College, China Three Gorges University, Yichang, Hubei, China

92 College of Traditional Chinese Medicine, Three Gorges University \& Yichang Hospital of 10 Traditional Chinese Medicine, Yichang, China

$11{ }^{3}$ Maternity and Child Health Care Hospital, Three Gorges University, Yichang, Hubei, China
13

14

Corresponding Author:

Wei Wang ${ }^{1}$

No.3 Shengli Avenue, Yichang, Hubei, 443000, China

Email address: doctor.vi@163.com

Xianyun $\mathrm{Fu}^{2}$

No.8 Daxue Avenue, Yichang, Hubei, 443000, China

Email address: dinnar1@163.com

\section{Abstract}

Background. Guizhi Fuling Wan (GZFLW) is a widely used classical Chinese herbal formulae prescribed for the treatment of endometriosis (EMs). This study aimed to predict the key targets and mechanisms of GZFLW in the treatment of EMs by network pharmacology and molecular docking.

Methods. Firstly, related compounds and targets of GZFLW were identified through the TCMSP, BATMAN-TCM, and CASC Database. Then, the EMs target database was built by GeneCards. 
28 The overlapping targets between GZFLW and EMs were screened out, and then data of the PPI

29

30

31

32

33

34

35

36

37

38

39

40

41

42

43

44

45

46

47

48

49

50

51

52

53

54

55

56

57

58

59

60

61

62

63

64

network was obtained by the STRING database to analyze the interrelationship of these targets.

Furthermore, a topological analysis was performed to screen the hub targets. After that, molecular docking technology was used to confirm the binding degree of the main active compounds and hub targets. Then, the DAVID database and Metascape database were used for GO and KEGG enrichment analysis. Finally, immortalized human endometrial stromal cells were cultured for further analysis and experimental verification. Cell proliferation was assessed by methyl thiazolyl tetrazolium (MTT) colorimetric assay, protein levels were determined by Western blotting, and apoptosis was detected by AnnexinV/PI staining using flow cytometry.

Results. A total of 89 GZFLW compounds and 284 targets were collected. One hundred one matching targets were picked out as the correlative targets of GZFLW in treating EMs. Among these, 25 significant hub targets were recognized by the PPI network. Coincidently, molecular docking simulation indicated that the hub targets had a good bonding activity with most active compounds (69.71\%). Furthermore, 116 items, including the inflammatory reaction, RNA polymerase, DNA transcription, growth factor activity, and steroid-binding, were selected by GO enrichment analysis. Moreover, the KEGG enrichment analysis results included 100 pathways focused on the AGE-RAGE pathway, HIF pathway, PI3K Akt pathway, MAPK pathway, and TP53 pathway, which exposed the potential mechanisms of GZFLW in treating EMs. What's more, MTT colorimetric assay indicated that the cell proliferation could be inhibited by GZFLW. And compared with the control group, the protein levels of P53, BAX, and caspase 3 in drug groups were all increased in Western blotting results. The results of flow cytometry showed that the percentage of apoptotic in the GZFLW group was significantly higher than that in the control group.

Conclusion. Through the exploration of network pharmacology and molecular docking technology, GZFLW has a therapeutic effect on EMs through the multi-target mechanism. This study provided a good foundation for further experimental research.

\section{Introduction}

Endometriosis (EMs), a common painful gynecological disease, occurs in $10 \%$ women of childbearing age (Reid et al. 2019). The most frequent symptoms of EMs include dysmenorrhea, chronic pelvic pain, and infertility (Bruun et al. 2018; Lalani et al. 2018). The incidence of developing many comorbidities, including irritable bowel syndrome, constipation, ovarian cancer, endometrial cancer, is significantly higher among EMs patients comparing to the general population (Schomacker et al. 2018). Although current therapies, including surgery, nonsteroidal anti-inflammatory drugs (NSAIDs), hormone treatments, and so on, could relief some significant symptoms of EMs, these treatments have also led to some side effects and a high recurrence rate (Prefumo \& Rossi 2018; Rabinerson et al. 2018). The patient's quality of life declines, and half of them are not satisfied with the available medical support (Verket et al.

Peer) reviewing PDF | (2020:06:49732:2:0:NEW 9 Feb 2021) 
65

66

67

68

69

70

71

72

73

74

75

76

77

78

79

80

81

82

83

84

85

86

87

88

89

90

91

92

93

94

95

96

97

98

99

100

101

2018). Therefore, it is still necessary to find novel and useful treatment methods (Lukas et al. 2018).

Historical Chinese medical texts have documented the use of the traditional Chinese herbal formula Guizhi Fuling Wan (GZFLW) for the EMs-like symptoms such as dysmenorrhea since the late Eastern Han Dynasty (200-210AD), and it is still widely used today for the treatment of EMs (Li et al. 2018; Wu et al. 2015; Zhao 2016) due to excellent therapeutic effect, low side effects (Wang et al. 2018), and safety. GZFLW consists of the original powder of five natural plants, including Cinnamon Twig (CT), Poria Cocos (PC), Cortex Moutan (CM), Radix Paeoniae Rubra (RPR), and Peach Kernel (PK). Animal experiments have confirmed that GZFLW could relieve dysmenorrhea effectively (Lang et al. 2018; Yang 2019). Nevertheless, the complex pathogenesis of EMs and the multiplex mechanism of GZFLW remains unclear. The principles and mechanisms by which GZFLW is useful for treating EMs need to be uncovered.

The holistic treatment of traditional Chinese medicine (TCM) has attracted more and more attention. The mechanism of drug therapy has shifted from single target to multiple interacting targets mediated by multiple compounds. Nowadays, the extensive application of network pharmacology and molecular docking provides a more effective method for the research and evaluation of the multi-target effect of multi-component drugs on diseases, which can reveal the mechanism of TCM treatment from a holistic perspective (Hopkins 2008). In the progress of bioinformatics and pharmacy, network pharmacology has become a capable vehicle to reveal the compatibility mechanism of TCM prescription (Fang et al. 2017; Ming et al. 2017; Zhao et al. 2015). An increasing number of researches have used them to analyze the possible molecular mechanisms of TCMs. This systematic research conception is consistent with the holistic theory and the synergistic mechanism of TCM (Lang et al. 2018). However, the possible mechanism of GZFLW treating EMs has not been systematically studied by network pharmacology.

Therefore, in this study, we screened multiple databases to find the active components of GZFLW and its possible targets for treating EMs. Target genes of GZFLW and EMs were matched to obtain overlapping results. PPI network data was obtained through the STRING database, and the hub targets were screened through topology analysis. After that, molecular docking technology was used to screen and verify the binding degree of the main active compounds and hub targets. Finally, the DAVID database and Metascape database were used for gene ontology (GO) and Kyoto Encyclopaedia of Genes and Genomes (KEGG) enrichment analysis. GO is generally used to describe the genes function and genes relationships (Ashburner et al. 2000), and KEGG is used for the enrichment of functions and signaling pathways (Kanehis \& Goto 2000). The vital active ingredients and mechanism of GZFLW in the treatment of EMs were confirmed by molecular docking technology. The workflow is shown in Figure 1.

\section{Materials \& Methods}

\section{Chemical ingredients database building}

Peer) reviewing PDF | (2020:06:49732:2:0:NEW 9 Feb 2021) 
102

103

104

105

106

107

108

109

110

111

112

113

114

115

116

117

118

119

120

121

122

123

124

125

126

127

128

129

130

131

132

133

134

135

136

137

138

139

140

141

To collect the chemical ingredients of the five herbs contained in GZFLW, the Traditional Chinese Medicine Systems Pharmacology Database ( $\mathrm{Ru}$ et al. 2014) (TCMSP, http://sp.nwu.edu.cn/tcmsp.php), the Bioinformatics Analysis Tool for Molecular Mechanism of Traditional Chinese Medicine (Liu et al. 2016) (BATMAN-TCM, http://bionet.ncpsb.org/batman-tcm/index.php ) and Chinese Academy of Sciences Chemistry Database (Wang et al. 2015) (CASC, http://www.organchem.csdb.cn/scdb/default.htm ) were used, which are both bioinformatics analysis tools for the main components of TCM. Four hundred ninety-four herbal ingredients were screened out.

\section{Active ingredients screening}

According to the characteristics of absorption, distribution, metabolism, and excretion (ADME) of drug, oral bioavailability (OB), and drug-likeness (DL) were used as screening indexes. OB is the percentage of oral drugs absorbed into the bloodstream, which is a frequently-used pharmacokinetic parameter. The OB was obtained using the OBioavaill.1 software, which covers 805 different drug and drug-like molecules (Liu et al. 2013). OB is a crucial indicator to judge whether the active ingredient can become a feasible therapeutic molecule (Xu et al. 2012). DL is a parameter to measure the ADME of drug molecules, which could help optimize pharmacokinetic and pharmaceutical properties. The DL threshold is 0.18 (Liu et al. 2013), which depends on the average of Drugbank (https://www.drugbank.ca/). DL is often used to select the active ingredients with "drug-like" properties in TCM composition (Tao et al. 2013). The OB and DL indices of all the related ingredients are presented in the TCMSP. In this process, those ingredients with $\mathrm{OB} \geqq 30 \%$ and $\mathrm{DL} \geqq 0.18$ were chosen as the potential effect components for the next step.

\section{GZFLW targets prediction}

The active compounds' targets in GZFLW were obtained from TCMSP, BATMAN-TCM, and CASC Database, with the species limited as "Homo sapiens".

\section{Endometriosis therapeutic targets database building}

Related targets of EMs were screened out by the GeneCards Database (Stelzer et al. 2016) (https://www.genecards.org/). Gene card databases are often used to predict genetic information related to human diseases. We searched the databases with the keyword "endometriosis" to obtain targets. Finally, the targets of GZFLW active ingredients were matched with the therapeutic targets of EMs. The overlapping targets were chosen as the potential targets of GZFLW in the treatment of EMs. These targets were then uploaded to STRING Database (Szklarczyk et al. 2017) (https://string-db.rog/) to obtain the interactions of the screened targets with the confidence $\geqq 0.7$ and the result of protein-protein interaction (PPI).

\section{Network topological feature set definitions}

We chose two parameters to evaluate the topological characteristics: "Degree" reflects the number of other nodes interacting with this node; "Betweenness Centrality" (BC) is measured by the percentage of all shortest paths. The nodes with high betweenness can significantly impact the net by controlling the information transmitted between other nodes. The node parameters are positively correlated with their topology importance in the network. 
142 Molecular docking of hub targets and active ingredients

143 Molecular docking is a computational tool to predict the binding ability and connection type of 144 proteins and ligands. It can calculate and predict the conformation and direction of ligands at 145 active protein sites. AutoDock1.5.6 (http://autodock.scripps.edu/) was a molecular docking 146 software that could be used to dock the hub targets and active ingredients based on network 147 pharmacology. In this docking process, the 3D structure of thirteen hub targets were retrieved 148 from RCSB Protein Data Bank (PDB) (http://www.rcsb.org/): AKT1 (PDB ID: 4EKL, 6S9W), 149 TNF (PDB ID: 2E7A, 2ZJC, 2AZ5), TP53 (PDB ID: 4MZI, 6FF9), VEGFA (PDB ID: 4WPB, 150 3QTK, 4QAF), MAPK1 (PDB ID: 4G6N, 1WZY), MMP9 (PDB ID: 6ESM, 4WZV), JUN (PDB 151 ID: 5T01, 1T2K), MAPK8 (PDB ID: 3VUK, 4L7F), INS (PDB ID: 4AJX, 4CY7), EGF (PDB 152 ID: 1JL9,), IL6 (PDB ID: 4O9H, 1ALU), PTGS2 (PDB ID: 5F19), and FOS (PDB ID: 1FOS); the 3D shapes of active compounds were provided from ZINC Database (http://zinc.docking.org/) and PubChem Database (https://pubchem.ncbi.nlm.nih.gov/). Binding energy was used as docking score to evaluate the protein-ligand binding potential of molecular docking. Among them, those results with value $\leq-5$ were selected and considered to have moderate binding potential and tight combination.

\section{Enrichment analysis}

160

We used the DAVID (DAVID, https://david.ncifcrf.gov/) (Huang et al. 2008) for GO enrichment analysis, including biological process (BP), molecular function (MF) and cellular component (CC). KEGG enrichment analysis was performed using the Metascape database to obtain potential target-pathways(Zhou et al. 2019) (http://metascape.org/).

163

\section{Network construction}

165

Networks, including the hub target-compound and target-pathway, were then constructed. All networks were built using Cytoscape 3.7.2 (http://www.cytoscape.org/) (Shannon et al. 2003), an open-source software platform for visualization and data analysis of complex networks.

\section{Primary endometrial stromal cells culture}

169

HEM15a cells were cultured in DMEM containing 10\% FBS and 1\% penicillin/streptomycin at $37^{\circ} \mathrm{C}$ in the cell incubator with a humid atmosphere containing $5 \% \mathrm{CO} 2$. After that, the vimentin staining of cells was identified by immunofluorescence. MTT colorimetric assay

The hEM15a cells were seeded in a 96-well plate and divided into the control group and the drug group. After incubation at $37^{\circ} \mathrm{C}$ for $48 \mathrm{~h}$ and reacted with GZFLW of different concentrations, hEM15a cells were reacted with $10 \mu \mathrm{L}$ MTT solution. Finally, $150 \mu \mathrm{L}$ dimethylsulfoxide (DMSO) was added after reacting for $4 \mathrm{~h}$. The optical absorbance was detected at $568 \mathrm{~nm}$ by a plate reader.

\section{Western blotting}

The hEM15a cells were splitted in RIPA buffer (Beyotime) added with 10 $\mu$ PMSF (Aladdin) and phosphatase inhibitors (Beyotime) to extract total proteins. After that, equivalent amounts of proteins were resolved by poly acrylamide gel electrophoresis (PAGE) and transferred to polyvinylidene fluoride (PVDF) membranes (Millipore, Massachusetts, USA). Then, TBST with 
182

183

184

185

186

187

188

189

190

191

192

193

194

195

196

197

198

199

200

201

202

203

204

205

206

207

208

209

210

211

212

213

214

215

216

217

218

219

220

5\% skim milk was adopted for blocking. Afterward, the membranes were reacted with primary antibodies against GAPDH (1:1000, AB-P-R 001, Xianzhi, Hangzhou, China), BAX (1:1000, Ab32503, Abcam, Britain), P53 (1:1000, 10442-1-AP, Sanying, Wuhan, China), and caspase3 (1:2000, Ab184787, Abcam), at $4{ }^{\circ} \mathrm{C}$ overnight. Subsequently, the matched secondary antibody was added to the membranes.

\section{Apoptosis analysis by flow cytometry}

The apoptosis of hEM15a cells was evaluated by Annexin V-FITC/PI apoptosis kit (KaiJi, Nanjing, China). The collected hEM15a cells, divided into normal group and GZFLW group, were resuspended in 500ul binding buffer and added with Annexin V-PI solution. Then, the hEM15a cells were incubated for 5 15 min without light. The percentage of apoptotic cells was then immediately detected on a flow cytometry (Beckmancoulter).

\section{Results}

\section{Active compounds of GZFLW}

By retrieving from the TCMSP, BATMAN-TCM, and CASC Database, there were 494 related ingredients of GZFLW in total, and there were 220 (44.5\%) of CT, 34 (6.9\%) of PC, 55 (11.1\%) of CM, 119 (24.1\%) of RPR, 66 (13.3\%) of PK. With OB $\geqq 30 \%$ and DL $\geqq 0.18$ as indexes, 89 active ingredients were screened out (Table 1.), and the Herbs-Compounds network was constructed as Fig. 2.

\section{Target prediction analysis}

In this process, we collected 284 targets of 89 active ingredients, and there were 58 in CT, 48 in PC, 185 in CM, 104 in RPR, and 62 in PK. Via the keyword of "endometriosis," the therapeutic targets of EMs were obtained from GeneCard Database with a total of 1350. One hundred one overlapping targets were then obtained as the related targets of GZFLW in the treatment of EMs (Fig.3.).

The data of the PPI network of those 101 targets were subsequently obtained in STRING Database. With confidence $\geqq 0.7$, there were 95 nodes and 751 edges in total. Taking two essential parameters of "degree" and "betweenness" as screening indexes, the topological analysis of targets mentioned above was performed. Targets more significant than or equal to the median are used as hub targets for GZFLW against EMs. The screened thresholds were degree $\geqq 11.5$ and betweenness $\geqq 0.007$, and the results were 25 hub nodes with 234 edges, including IL6, JUN, TNF, MAPK1, TP53, EGF, MAPK8, MMP9, VEGFA, AKT1, INS, FOS, ICAM1, PTGS2, CCL2, CCND1, EGFR, IL1B, MYC, IL10, PTEN, ESR1, PPARG, RELA and MMP2 (Table 2.). When the 25 significant hub nodes and other 70 nodes were distributed with "degree" and "betweenness", the network of 95 nodes was built as Fig.4.

Based on the 25 key targets and related 29 active ingredients, we further established the network of Hub nodes-Compounds (Fig.5.). Among them, quercetin (MOL000098) is associated with 21 hub targets, and kaempferol (MOL000422) interrelates to 7 key targets. Besides, baicalein (MOL002714) is related to 6 key targets, while pachymic acid (MOL000289), ellagic acid (MOL001002), and taxifolin (MOL004576) act on three key targets respectively. 
221

222

223

224

225

226

227

228

229

230

231

232

233

234

235

236

237

238

239

240

241

242

243

244

245

246

247

248

249

250

251

252

253

254

255

256

257

258

259

260

\section{Molecular Docking}

Thirteen hub targets with top degrees of GZFLW were identified with seven active compounds by AutoDock. There were 122 results (69.71\%) of them had a moderate binding potential, which indicated that active ingredients of GZFLW could well bind to the targets for the treatment of EMs (Table 3.). The ligands are mainly linked with corresponding proteins and critical amino acids around them in the form of hydrogen bonds (Fig. 6.).

\section{GO and KEGG pathway enrichment analysis}

With the database of DAVID and Metascape, the enrichment analysis on 101 targets was performed and resulted in 116 GO items and 100 KEGG pathways.

\section{GO enrichment analysis}

After 116 items were sorted in descending order based on P-value, the first eight items of three parts, BP, MF, and CC, were selected (Fig.7.). In the aspect of BP, we mainly had: inflammatory response (GO:0006954), positive regulation of transcription from RNA polymerase II promoter (GO:0045944), transcription DNA-templated (GO:0006351), lipopolysaccharide-mediated signaling pathway (GO:0031663), positive regulation of cell division (GO:0051781), negative regulation of growth of symbiont in host (GO:0044130), response to toxic substance (GO:0009636) and positive regulation of cytokine secretion (GO:0050715); in the part of MF, we obtained heme binding (GO:0020037), sequence-specific DNA binding (GO:0043565), transcription factor activity sequence-specific DNA binding (GO:0003700), growth factor activity (GO:0008083), identical protein binding (GO:0042802), protein homodimerization activity (GO:0042803), cytokine activity (GO:0005125) and steroid binding (GO:0005496); in the aspect of CC, there were extracellular space (GO:0005615), nucleus (GO:00056340), cytosol (GO:0005829), membrane raft (GO:0045121), apical plasma (GO:0016324), external side of plasma membrane (GO:0009897), endoplasmic reticulum membrane (GO:0005789) and extrinsic component of external side of plasma membrane (GO:0031232). Based on the above three aspects, it is possible that the mechanism of GZFLW in treating EMs was the result of multi-pathway synergy.

\section{KEGG pathway enrichment analysis}

To further illustrate the potential mechanism of GZFLW in the treatment of EMs, we performed the KEGG pathway enrichment analysis on 101 targets. We selected the top 20 pathways based on the $P$-value, such as the AGE-RAGE signaling pathway(hsa04933), HIF-1 signaling pathway (hsa04066), PI3K-Akt signaling pathway (hsa04151), MAPK signaling pathway (hsa04010), EGFR tyrosine kinase inhibitor resistance (hsa01521) (Fig.8.). Then, we constructed the Target-Pathway Network to intuitively reveal the relationship between the hub targets and pathways (Fig.9.).

\section{Primary endometrial stromal cells culture}

The primary endometrial stromal cells were spindle-shaped or star-shaped with large and round nuclei, and proliferative fibrous tissue could be seen. After that, the immunofluorescence was used to identified the vimentin staining of cells in different groups (Fig.10.).

\section{MTT colorimetric assay}


261

262

263

264

265

266

267

268

269

270

271

272

273

274

275

276

277

278

279

280

281

282

283

284

285

286

287

288

289

290

291

292

293

294

In comparison with the control group, in addition to $0.5 \mathrm{mg} / \mathrm{ml}$, the other different doses of treatment groups $(1 \mathrm{mg} / \mathrm{ml}, 3 \mathrm{mg} / \mathrm{ml}, 5 \mathrm{mg} / \mathrm{ml}, 10 \mathrm{mg} / \mathrm{ml}, 20 \mathrm{mg} / \mathrm{ml})$ had significant inhibitory effect on the proliferation of endometrial stromal cells; ( $p<0.05,0.01$ or 0.001 ; Fig.11.).

\section{Western blotting}

Western blot analysis revealed that compared to the control groups, drug treatment increased the protein levels of P53, BAX, and caspase 3 ( $p<0.05$ or 0.01 ) in endometriotic lesions (Fig. 12.).

\section{Apoptosis analysis by flow cytometry}

Compared with the control group, the percentage of apoptotic cells in the GZFLW group with the dose of $4 \mathrm{mg}$ increased significantly (Fig. 13.).

\section{Discussion}

As we all know, TCM is characterized by multiple components, multiple targets, and multiple pathways in the treatment of diseases (Lang et al. 2018). Because of the complex composition, TCM's clinical and pharmacological research is often difficult to carry out. Network pharmacology is a systematic research method, suitable for the "multi-component, multi-target, multi-pathway" synergistic characteristics of TCM. In this study, we are the first to explore the possible therapeutic mechanism of GZFLW on EMs through network pharmacology and molecular docking methods, to provide directions and ideas for further experimental research.

\section{The Major Active Ingredients of GZFLW}

Based on the Hub nodes-Compound Network, we found a few hub compounds: quercetin and kaempferol contained in CM, taxifolin contained in CT, pachymic acid contained in PC, ellagic acid, baicalein, and paeoniflorin contained in RPR, campesterol, hederagenin contained in PK, $\beta$ sitosterol, contained in CM, RPR and PK. Among them, quercetin, kaempferol, taxifolin, and baicalein are flavonoids that have shown potent anti-EMs activity by effectively relieving symptoms and inhibiting levels of CA-125 (Signorile et al. 2018). Quercetin and kaempferol can significantly reduce the ailing area of the endometrium via anti-proliferation and antiinflammatory effects (Park et al. 2019) in EMs mice (Ilhan et al. 2020). Previous studies have demonstrated that phytosterols, including $\beta$-sitosterol and campesterol, contributed to the regression of EMs (Ilhan et al. 2019). Baicalin has also been reported to treat EMs by reducing the activity of endometrial stromal cell (ESC) (Jin et al. 2017), which provided evidence for our results. In conclusion, we speculate that these above ingredients are the potential material basis of GZFLW in treating EMs.

It is mainly indicated that the pathways may be associated with treating EMs of GZFLW were regarding the immune response, apoptosis and proliferation, oxidative stress, and angiogenesis.

Inflammatory response 
295

296

297

298

299

300

301

302

303

304

305

306

307

308

309

310

311

312

313

314

315

316

317

318

319

320

321

322

323

324

325

326

327

328

329

330

331

332

333

The differentially expressed genes are related to inflammation in EMs (Ahn et al. 2016), which suggest its role in the course of EMs. Prostaglandin-endoperoxide synthase (PTGS2), also known as COX-2, is related to the pain and infertility of EMs and the PTGS2/ PGE2 axis is considered as the critical target during EMs (Li et al. 2020). TNF, IL1- $\beta$ are the other hub targets in this study. The immunoreactivity of which induces inflammation in the peritoneal fluid of EMs through activating NF-kappa B and mitogen-activated protein kinase (MAPKs) signaling pathways (Kralickova et al. 2018). To some extents, components such as hederagenin, campesterol, $\beta$-sitosterol, pachymic acid, quercetin, and taxifolin in GZFLW inhibit the expression of inflammatory factors to influence the whole inflammatory response. Previous studies have demonstrated that hederagenin inhibits the expression of inflammatory factors, including TNF, IL1, IL6, COX-2 via MAPKs and NF-kappaB pathway (Akhtar et al. 2019; Kim et al. 2017; Lu et al. 2015). Campesterol, $\beta$-sitosterol, known as sterols, can reduce the levels of TNF- $\alpha$ and IL of peritoneal fluid in the EMs mice (Ilhan et al. 2019). Flavonoids have a similar effect. Studies have experimentally proved that quercetin has an anti-inflammatory effect on the EMs (Park et al. 2019). Coincidentally, the docking result confirmed all the above, which also provides certain credibility for our results that GZFLW possibly worked against inflammatory in a multi-ingredient way.

\section{Apoptosis and proliferation}

The cell apoptosis's inhibition and concomitant cell excessive proliferation play some important roles in the development of EMs. We think that the expression and regulation of the Jun and Fos genes are among the key factors in cell proliferation and apoptosis. It is found that high expression of c-Jun related to the proliferation of EMs cells via the JNK / c-Jun signaling pathway ( $\mathrm{Yu}$ et al. 2018). TP53 is another critical tumor suppressor gene in our study; its regulative effect on the cell cycle plays a crucial role in obtaining proliferation activity for ESC of EMs (Hirakawa et al. 2016). It is investigated that the polymorphisms of TP53 may be involved in high risk of the generation of EMs (Hussain et al. 2018). Epidermal growth factor (EGF) also plays a vital role in regulating cell growth, proliferation, and differentiation. Previous studies have found that the EGF expression in patients with severe EMs significantly increased (Chatterjee et al. 2018). Furthermore, there are researches shown that COX-2 expression regulated by the PTGS2 gene can activate MAPKs, which then subsequently activate some transcription factors and protein kinases, ultimately promoting the proliferation of cells.

GZFLW's regulative effect on cell proliferation and apoptosis in EMs is possibly achieved by regulating flavonoids on the expression of PTGS2 (da Silva et al. 2020), which further regulates the COX-2/PGE2 axis (Takaoka et al. 2018). Besides, components such as flavonoids, quercetin in GZFLW affect the proliferation and apoptosis in EMs by inhibiting the ERK1/2, P38 MAPK, and AKT signaling pathway (Park et al. 2019). Also, baicalin can affect the activity of ESC by apoptosis inhibition via the NF-kappa B signaling pathway (Jin et al. 2017). Furthermore, the direct or indirect inhibition of natural triterpenoids on proliferation is possibly another essential component of the regulation of cell apoptosis (Chen et al. 2019).

Peer] reviewing PDF | (2020:06:49732:2:0:NEW 9 Feb 2021) 
334

335

336

337

338

339

340

341

342

343

344

345

346

347

348

349

350

351

352

353

354

355

356

357

358

359

360

361

362

363

364

365

366

367

368

369

370

It is mentioning that all the primary active ingredients except paeoniflorin had a high docking score with EGF in our docking results. Besides, three active ingredients (hederagenin, phytosterol, and pachymic acid) had tight combinations with Jun, FOS, and PTGS2, and the other three active ingredients (pachymic acid, taxifolin, and hederagenin) had strong putative interaction with P53, which demonstrates that GZFLW is likely to affect on proliferation and apoptosis through a multi-ingredient synergistic way.

To verify the effect of GZFLW on EMs, we conducted in vitro experiments. The results of MTT and flow cytometry confirmed that GZFLW can effectively promote the apoptosis of ectopic stromal cells in EMs. In the treatment group, the expression levels of P53, Capase3 and Bax were significantly up-regulated, suggesting that the therapeutic effect of GZFLW may be related to the regulation of P53 pathway and apoptosis pathway.

\section{Oxidative stress}

More and more evidence has shown that the pathophysiological mechanism of EMs is complicated, and hypoxia plays an essential role in EMs damage (Wu et al. 2019). The AGE/RAGE and HIF-1alpha signaling pathway were speculated as probable mechanisms of GZFLW against EMs from KEGG results. Previous studies have demonstrated that high expression of RAGE under hypoxia environment increased the accumulation of ROS in peritoneal fluid (Polak et al. 2018). Eventually, contribute to the cell invasion ability in EMs (Seguella et al. 2019). It is also found that high expression of HIF-1 $\alpha$ in the serum of EMs aggravated the severity of dysmenorrheal (Zhang et al. 2018).

Fortunately, triterpenoids and flavonoids including hederagenin, $\beta$-sitosterol (Adebiyi et al. 2019; Ponnulakshmi et al. 2019), campesterol (Alvarez-Sala et al. 2018; Eom et al. 2017; Ho et al. 2016; Ogunlaja et al. 2016; Shamloo et al. 2017; Toiu et al. 2019; Weingartner et al. 2017), taxifolin, and pachymic acid were reported to be the natural antioxidants, which could reduce oxidative stress by inhibiting RAGE expression to a certain extent (Song et al. 2017) via MAPK, and PI3K/Akt pathways (Wen et al. 2018). The molecular docking results showed that triterpenoids and flavonoids have tight combinations with the hub targets AKT1, MAPK1, and MAPK8. However, further experiments are needed to confirm whether GZFLW worked against EMs by affecting AGE/RAGE and HIF-1 $\alpha$ via MAPK and PI3K/Akt pathways.

\section{Angiogenesis}

Hypoxia and inflammatory, as an essential characteristic of EMs microenvironment, can stimulate the transcription of hypoxia-inducible factor HIF (Lin et al. 2018) and inflammatoryinducible factor COX-2 gene, consequently activate the angiogenesis through the $\mathrm{PI} 3 \mathrm{~K} / \mathrm{mTOR}$ and MAPK pathways. HIF-1 $\alpha$ / VEGF expression in serum and endometrium has been reported to have a relationship with the stage of EMs and the severity of dysmenorrheal (Zhang et al. 2018). Studies confirmed that some plant inhibitors of COX-2 were able to treat EMs and inhibited ESC's angiogenesis by regulating HIF-1 $\alpha$ /VEGF in mice models (Li et al. 2020).

Peer) reviewing PDF | (2020:06:49732:2:0:NEW 9 Feb 2021) 
371 It is worth mentioning that triterpenoids ( $\beta$-sitosterol, campesterol, hederagenin) and 372 flavonoids (taxifolin) in GZFLW were detected in our study, they could have a modest effect on 373 inhibiting VEGF in EMs, which have been confirmed by experiments and in line with our 374 molecular docking results (Ilhan et al. 2019). Previous studies have also demonstrated that 375 paeoniflorin could inhibit angiogenesis via the HIF-1 $\alpha$ / VEGF pathway (Song et al. 2017; Zhou 376 et al. 2018).

\section{Conclusions}

379

380

381

382

383

384

385

386

387

388

389

390

391

392

393

394

395

396

397

398

399

400

401

402

403

404

405

406

In this study, network pharmacology and molecular docking were utilized for exploring the potential effects of GZFLW in anti-EMs, mainly focusing on four aspects: the suppression of inflammatory response, the regulating of apoptosis and proliferation, the reduction of the oxidative stress, and the inhibition of angiogenesis. Among them, the pro-apoptotic effect was confirmed by our in vitro experiments. In these crucial biological functions, triterpenoids and flavonoids contained in the GZFLW were considered that those might be the critical active ingredients involved in the potential therapy mechanisms.

Our study had several limitations. Firstly, to some extent, network pharmacologic analysis can help to predict the possible molecular mechanism of GZFLW in treating EMs. However, we can only speculate but not confirm if such mechanisms have an impact because, in evidence-based medicine, the conclusion can not be determined until subsequent biological experiments. Secondly, the data set of the GZFLW related compounds and targets were identified through the TCMSP, BATMAN-TCM, and CASC Database, and GeneCards built the EMs target database. Choosing different databases may lead to the study biases. Moreover, because all the databases we use are based on existing research results, this may limit the discovery of new targets related to EMs treatment.

In conclusion, our study reveals that GZFLW may have a multiple effect on EMs. Although the mechanism remains confirmed by further experiments, the relevant targets and signaling pathways for GZFLW against EMs have been preliminarily studied and systematically summarized.

\section{References}

\section{References}

Adebiyi OE, Olayemi FO, Olopade JO, and Tan NH. 2019. Beta-sitosterol enhances motor coordination, attenuates memory loss and demyelination in a vanadium-induced model of experimental neurotoxicity. Pathophysiology 26:21-29.

Ahn SH, Khalaj K, Young SL, Lessey BA, Koti M, and Tayade C. 2016. Immune-inflammation gene signatures in endometriosis patients. Fertil Steril 106:1420-1431.

Peer] reviewing PDF | (2020:06:49732:2:0:NEW 9 Feb 2021) 
407

408

409

410

411

412

413

414

415

416

417

418

419

420

421

422

423

424

425

426

427

428

429

430

431

432

433

434

435

436

437

438

439

440

441

442

443

444

445

446

447

448

449

450

451

452

453

454

Akhtar M, Shaukat A, Zahoor A, Chen Y, Wang Y, Yang M, Umar T, Guo M, and Deng G. 2019. Antiinflammatory effects of Hederacoside-C on Staphylococcus aureus induced inflammation via TLRs and their downstream signal pathway in vivo and in vitro. Microb Pathog 137:103767.

Alvarez-Sala A, Lopez-Garcia G, Attanzio A, Tesoriere L, Cilla A, Barbera R, and Alegria A. 2018. Effects of Plant Sterols or beta-Cryptoxanthin at Physiological Serum Concentrations on Suicidal Erythrocyte Death. Journal of Agricultural \& Food Chemistry 66:1157-1166.

Ashburner M, Ball CA, Blake JA, Botein D, Butler H, Cherry JM, Davis AP, Dolinski K, Dwight SS, Eppig JT, Harris MA, Hill DP, Issel-Tarver L, Kasarskis A, Lewis S, Matese JC, Richardson JE, Ringwald M, Rubin GM, and Sherlock G. 2000. Gene Ontology: tool for the unification of biology. Nature Genetics 25:25-29.

Bruun MR, Arendt LH, Forman A, and Ramlau-Hansen CH. 2018. Endometriosis and adenomyosis are associated with increased risk of preterm delivery and a small-for-gestational-age child: a systematic review and meta-analysis. Acta Obstet Gynecol Scand 97:1073-1090. 10.1111/aogs.13364

Chatterjee K, Jana S, DasMahapatra P, and Swarnakar S. 2018. EGFR-mediated matrix metalloproteinase-7 up-regulation promotes epithelial-mesenchymal transition via ERK1-AP1 axis during ovarian endometriosis progression. FASEB J 32:4560-4572. 10.1096/fj.201701382RR

Chen Z, Huang KY, Ling Y, Goto M, Duan HQ, Tong XH, Liu YL, Cheng YY, Morris-Natschke SL, Yang PC, Yang SL, and Lee KH. 2019. Discovery of an Oleanolic Acid/Hederagenin-Nitric Oxide Donor Hybrid as an EGFR Tyrosine Kinase Inhibitor for Non-Small-Cell Lung Cancer. J Nat Prod 88:30653073.

da Silva $A B$, Cerqueira Coelho PL, das Neves Oliveira M, Oliveira JL, Oliveira Amparo JA, da Silva KC, Soares JRP, Pitanga BPS, Dos Santos Souza C, de Faria Lopes GP, da Silva VDA, de Fatima Dias Costa M, Junier MP, Chneiweiss H, Moura-Neto V, and Costa SL. 2020. The flavonoid rutin and its aglycone quercetin modulate the microglia inflammatory profile improving antiglioma activity. Brain Behav Immun 85:170-185. 10.1016/j.bbi.2019.05.003

Eom MR, Weon JB, Jung YS, Ryu GH, Yang WS, and Ma CJ. 2017. Neuroprotective compounds from Reynoutria sachalinensis. Archives of Pharmacal Research 40:704-712.

Fang HY, Zeng HW, Lin LM, Chen X, Shen XN, Fu P, Lv C, Liu Q, Liu RH, Zhang WD, and Zhao J. 2017. A network-based method for mechanistic investigation of Shexiang Baoxin Pill's treatment of cardiovascular diseases. Sci Rep 7:43632.

Hirakawa T, Nasu K, Abe W, Aoyagi Y, Okamoto M, Kai K, Takebayashi K, and Narahara H. 2016. miR-503, a microRNA epigenetically repressed in endometriosis, induces apoptosis and cell-cycle arrest and inhibits cell proliferation, angiogenesis, and contractility of human ovarian endometriotic stromal cells. Hum Reprod 31:2587-2597. 10.1093/humrep/dew217

Ho XL, Liu JJ, and Loke WM. 2016. Plant sterol-enriched soy milk consumption modulates 5-lipoxygenase, 12-lipoxygenase, and myeloperoxidase activities in healthy adults - a randomized-controlled trial. Free Radical Research Communications 50:1396-1407.

Hopkins AL. 2008. Network pharmacology: the next paradigm in drug discovery. Nat Chem Biol 4:682690.

Huang DW, Sherman BT, and Lempicki RA. 2008. Systematic and integrative analysis of large gene lists using DAVID bioinformatics resources. Nat Protoc 4:44.

Hussain R, Khaliq S, Raza SM, Khaliq S, and Lone K. 2018. Association of TP53 codon 72 polymorphism in women suffering from endometriosis from Lahore, Pakistan. J Pak Med Assoc 68:224-230.

Ilhan M, Ali Z, Khan IA, and Tastan H. 2020. The regression of endometriosis with glycosylated flavonoids isolated from Melilotus officinalis (L.) Pall. in an endometriosis rat model. Taiwan J Obstet Gynecol 59:211-219.

Ilhan M, Ali Z, Khan IA, Tastan H, and Akkol EK. 2019. Promising activity of Anthemis austriaca Jacq. on

Peer] reviewing PDF | (2020:06:49732:2:0:NEW 9 Feb 2021) 
455

456

457

458

459

460

461

462

463

464

465

466

467

468

469

470

471

472

473

474

475

476

477

478

479

480

481

482

483

484

485

486

487

488

489

490

491

492

493

494

495

496

497

498

499

500

501

502

the endometriosis rat model and isolation of its active constituents. Saudi Pharm J 27:889-899.

Jin Z, Huang J, and Zhu Z. 2017. Baicalein reduces endometriosis by suppressing the viability of human endometrial stromal cells through the nuclear factor-kappaB pathway in vitro. Exp Ther Med 14:2992-2998.

Kanehis M, and Goto S. 2000. KEGG: kyoto encyclopedia of genes and genomes. Nucleic Acids Research 28:27-30.

Kim GJ, Song DH, Yoo HS, Chung KH, Lee KJ, and An JH. 2017. Hederagenin Supplementation Alleviates the Pro-Inflammatory and Apoptotic Response to Alcohol in Rats. Nutrients 9.

Kralickova M, Fiala L, Losan P, Tomes P, and Vetvicka V. 2018. Altered Immunity in Endometriosis: What Came First? Immunol Invest 47:569-582.

Lalani S, Choudhry AJ, Firth B, Bacal V, Walker M, Wen SW, Singh S, Amath A, Hodge M, and Chen I. 2018. Endometriosis and adverse maternal, fetal and neonatal outcomes, a systematic review and meta-analysis. Hum Reprod 33:1854-1865. 10.1093/humrep/dey269

Lang L, Meng Z, Sun L, Xiao W, Zhao L, and Xiong Z. 2018. Intergrated metabonomic study of the effects of Guizhi Fuling capsule intervention on primary dysmenorrheal using RP-UPLC-MS complementary with HILIC-UPLC-MS technique. Biomed Chromatogr 32.

Li J, Zeng Z, Chang Y, Li M, Wu Q, Chen P, and Liang X. 2020. Suppressive Effects of Ursolic Acid on Human Endometriotic Stromal Cells Survival. Gynecol Obstet Invest 85:72-81.

Li X, Xu X, Ma H, Xu F, Tian C, and Hou W. 2018. Clinical and experimental research progress on Guizhi Fuling Pill of classical herbal formulae. Drug Eval Res 41:1724-1729.

Lin X, Dai Y, Xu W, Shi L, Jin X, Li C, Zhou F, Pan Y, Zhang Y, Lin X, and Zhang X. 2018. Hypoxia Promotes Ectopic Adhesion Ability of Endometrial Stromal Cells via TGF-beta1/Smad Signaling in Endometriosis. Endocrinology 159:1630-1641.

Liu H, Wang J, Zhou W, Wang Y, and Yang L. 2013. Systems approaches and polypharmacology for drug discovery from herbal medicines: An example using licorice. J Ethnopharmacol 146:773-793.

Liu Z, Guo F, Wang Y, Li C, Zhang X, Li H, Diao L, Gu J, Wang W, Li D, and He F. 2016. BATMAN-TCM: a Bioinformatics Analysis Tool for Molecular mechANism of Traditional Chinese Medicine. Sci Rep 6:21146.

Lu SH, Guan JH, Huang YL, Pan YW, Yang W, Lan H, Huang S, Hu J, and Zhao GP. 2015. Experimental Study of Antiatherosclerosis Effects with Hederagenin in Rats. Evid Based Complement Alternat Med 2015:456354.

Lukas I, Kohl-Schwartz A, Geraedts K, Rauchfuss M, Wolfler MM, Haberlin F, von Orelli S, Eberhard M, Imthurn B, Imesch P, and Leeners B. 2018. Satisfaction with medical support in women with endometriosis. PLoS One 13:e0208023. 10.1371/journal.pone.0208023

Ming L, Yan CL, Liu HX, Wang TY, Shi XH, Liu JP, Orgah J, Fan JW, Han JH, Wang XJ, and Zhu Y. 2017. Network pharmacology exploration reveals endothelial inflammation as a common mechanism for stroke and coronary artery disease treatment of Danhong injection. Sci Rep 7:15427.

Ogunlaja OO, Moodley R, Baijnath H, and Jonnalagadda SB. 2016. CHEMICAL CONSTITUENTS AND IN VITRO ANTIOXIDANT ACTIVITY OF CRUDE EXTRACTS AND COMPOUNDS FROM LEAVES AND STEM BARK OF FICUS BURTT-DAVYI. Acta Poloniae Pharmaceutica 73:1593-1600.

Park S, Lim W, Bazer FW, Whang KY, and Song G. 2019. Quercetin inhibits proliferation of endometriosis regulating cyclin D1 and its target microRNAs in vitro and in vivo. J Nutr Biochem 63:87-100.

Polak G, Barczynski B, Wertel I, Kwaniewski W, Bednarek W, Derewianka-Polak M, Frszczak K, Olajossy M, and Kotarski J. 2018. Disrupted iron metabolism in peritoneal fluid may induce oxidative stress in the peritoneal cavity of women with endometriosis. Ann Agric Environ Med 25:587-592.

Ponnulakshmi R, Shyamaladevi B, Vijayalakshmi P, and Selvaraj J. 2019. In silico and in vivo analysis to identify the antidiabetic activity of beta sitosterol in adipose tissue of high fat diet and sucrose induced type-2 diabetic experimental rats. Toxicol Mech Methods 29:276-290.

Peer] reviewing PDF | (2020:06:49732:2:0:NEW 9 Feb 2021) 
503

504

505

506

507

508

509

510

511

512

513

514

515

516

517

518

519

520

521

522

523

524

525

526

527

528

529

530

531

532

533

534

535

536

537

538

539

540

541

542

543

544

545

546

547

548

549

550

Prefumo F, and Rossi AC. 2018. Endometriosis, endometrioma, and ART results: Current understanding and recommended practices. Best Pract Res Clin Obstet Gynaecol 51:34-40. 10.1016/j.bpobgyn.2018.01.019

Rabinerson D, Hiersch L, and Gabbay-Ben-Ziv R. 2018. [Dysmenorrhea - Its Prevalence, Causes, Influence on the Affected Women and Possible Treatments]. Harefuah 157:91-94.

Reid R, Steel A, Wardle J, Mclntyre E, Harnett J, Foley H, and Adams J. 2019. The prevalence of selfreported diagnosed endometriosis in the Australian population: results from a nationallyrepresentative survey. BMC Res Notes 12:88. 10.1186/s13104-019-4114-6

Ru J, Li P, Wang J, Zhou W, Li B, Huang C, Li P, Guo Z, Tao W, Yang Y, Xu X, Li Y, Wang Y, and Yang L. 2014. TCMSP: a database of systems pharmacology for drug discovery from herbal medicines. $J$ Cheminf 6:13.

Schomacker ML, Hansen KE, Ramlau-Hansen CH, and Forman A. 2018. Is endometriosis associated with irritable bowel syndrome? A cross-sectional study. Eur J Obstet Gynecol Reprod Biol 231:65-69.

Seguella L, Capuano R, Pesce M, Annunziata G, Pesce M, Conno BD, Sarnelli G, Aurino L, and Esposito G. 2019. S100B Protein Stimulates Proliferation and Angiogenic Mediators Release through RAGE/pAkt/mTOR Pathway in Human Colon Adenocarcinoma Caco-2 Cells. Int J Mol Sci 20.

Shamloo M, Babawale EA, Furtado A, Henry RJ, Eck PK, and Jones PJH. 2017. Effects of genotype and temperature on accumulation of plant secondary metabolites in Canadian and Australian wheat grown under controlled environments. Scientific Reports 7:9133.

Shannon P, Markiel A, Ozier O, Baliga NS, Wang JT, Ramage D, Amin N, Schwikowski B, and Ideker T. 2003. Cytoscape: a software environment for integrated models of biomolecular interaction networks. Genome Res 13:2498-2504.

Signorile PG, Viceconte R, and Baldi A. 2018. Novel dietary supplement association reduces symptoms in endometriosis patients. J Cell Physiol 233:5920-5925.

Song S, Xiao X, Guo D, Mo L, Bu C, Ye W, Den Q, Liu S, and Yang X. 2017. Protective effects of Paeoniflorin against AOPP-induced oxidative injury in HUVECs by blocking the ROS-HIF1alpha/VEGF pathway. Phytomedicine 34:115-126.

Stelzer G, Rosen N, Plaschkes I, Zimmerman S, Twik M, Fishilevich S, Stein TI, Nudel R, Lieder I, Mazor Y, Kaplan S, Dahary D, Warshawsky D, Guan-Golan Y, Kohn A, Rappaport N, Safran M, and Lancet D. 2016. The genecards suite: from gene data mining to disease genome sequence analyses. Current Protocols in Bioinformatics 54:1 3031 - 313033.

Szklarczyk D, Morris JH, Cook H, Kuhn M, Wyder S, Simonovic M, Santos A, Doncheva NT, Roth A, Bork P, Jensen JJ, and Mering C. 2017. The STRING database in 2017: quality-controlled protein-protein association networks, made broadly accessible. Nucleic Acids Res 45:D362 - D368.

Takaoka O, Mori T, Ito F, Okimura H, Kataoka H, Tanaka Y, Koshiba A, Kusuki I, Shigehiro S, Amami T, and Kitawaki J. 2018. Daidzein-rich isoflavone aglycones inhibit cell growth and inflammation in endometriosis. J Steroid Biochem Mol Biol 181:125-132.

Tao W, Xu X, Wang X, Li B, Wang Y, Li Y, and Yang L. 2013. Network pharmacology-based prediction of the active ingredients and potential targets of Chinese herbal Radix Curcumae formula for application to cardiovascular disease. J Ethnopharmacol 145:1-10.

Toiu A, Mocan A, Vlase L, Parvu AE, Vodnar DC, Gheldiu AM, Moldovan C, and Oniga I. 2019. Comparative Phytochemical Profile, Antioxidant, Antimicrobial and In Vivo Anti-Inflammatory Activity of Different Extracts of Traditionally Used Romanian Ajuga genevensis L. and A. reptans L. (Lamiaceae). Molecules 24.

Verket NJ, Uhlig T, Sandvik L, Andersen MH, Tanbo TG, and Qvigstad E. 2018. Health-related quality of life in women with endometriosis, compared with the general population and women with rheumatoid arthritis. Acta Obstet Gynecol Scand 97:1339-1348. 10.1111/aogs.13427

Wang SS, Xu HY, Yan M, Wang XG, Shi Y, Huang B, Tang SH, Zhang Y, Li DF, Liang RX, and Yang HJ. 2015.

Peer] reviewing PDF | (2020:06:49732:2:0:NEW 9 Feb 2021) 
551

552

553

554

555

556

557

558

559

560

561

562

563

564

565

566

567

568

569

570

571

572

573

574

575

576

577

578

579

580

581

582

583

584

585

586

587

588
Characterization and rapid identification of chemical constituents of NaoXinTong capsules by UHPLC-linear ion trap/Orbitrap mass spectrometry. J Pharm Biomed Anal 111:104-118.

Wang W, Gao Y, Liu F, Wei R, and Xie Y. 2018. Post-marketing surveillance on Guizhi Fuling Jiaonang based on literature review. Chin J Chin Mater Med 43:820-832.

Weingartner O, Bogeski I, Kummerow C, Schirmer S, H., Vanmierlo T, Wagenpfeil G, Hoth M, Bohm M, Lutjohann D, and Laufs U. 2017. Plant sterol ester diet supplementation increases serum plant sterols and markers of cholesterol synthesis, but has no effect on total cholesterol levels. The Journal of steroid biochemistry and molecular biology 169:219-225.

Wen Z, Hou W, Wu W, Zhao Y, Dong X, Bai X, Peng L, and Song L. 2018. 6'-O-Galloylpaeoniflorin Attenuates Cerebral Ischemia Reperfusion-Induced Neuroinflammation and Oxidative Stress via $\mathrm{PI3K} /$ Akt/Nrf2 Activation. Oxidative Medicine and Cellular Longevity 2018:8678267.

Wu MH, Hsiao KY, and Tsai SJ. 2019. Hypoxia: The force of endometriosis. J Obstet Gynaecol Res 45:532541.

Wu X, Yang E, Sun Z, Piao C, He L, and Zhang S. 2015. Experimental research of "Guizhi Fuling Pills"for the treatment of dysmenorrhea of bloodstasis type. Shanghai J Tradit Chin Med 49:102-105.

Xu X, Zhang W, Huang C, Li Y, H. Y, Wang Y, Duan J, and Ling Y. 2012. A Novel Chemometric Method for the Prediction of Human Oral Bioavailability. Int J Mol Sci 13:6964-6982.

Yang Y. 2019. Systematic evaluation of Chinese patent medicine in the treatment of secondary dysmenorrhea (endometriosis, adenomyosis) Master. Beijing Univ Tradit Chin Med.

Yu J, Francisco AMC, Patel BG, Cline JM, Zou E, Berga SL, and Taylor RN. 2018. IL-1beta Stimulates BrainDerived Neurotrophic Factor Production in Eutopic Endometriosis Stromal Cell Cultures: A Model for Cytokine Regulation of Neuroangiogenesis. Am J Pathol 188:2281-2292. 10.1016/j.ajpath.2018.06.011

Zhang F, Liu XL, Wang W, Dong HL, Xia YF, Ruan LP, and Liu LP. 2018. Expression of MMIF, HIF-1alpha and VEGF in Serum and Endometrial Tissues of Patients with Endometriosis. Curr Med Sci 38:499-504.

Zhao F, Guochun L, Yang Y, Shi L, Xu L, and Yin L. 2015. A network pharmacology approach to determine active ingredients and rationality of herb combinations of modified-Simiaowan for treatment of gout. J Ethnopharmacol 168:1-16.

Zhao Y. 2016. Clinical study on Guizhi Fuling Pill in the treatment of gynecological diseases. J Hebei Tradit Chin Med Pharm 31:30-31.

Zhou J, Ding ZM, and Hardiman PJ. 2018. Understanding the Role of Gui-Zhi-Fu-Ling-Capsules (Chinese Medicine) for Treatment of Endometriosis in the Rat Model: Using NMR Based Metabolomics. Evidence-Based Complementary and Alternative Medicine 2018:9864963.

Zhou Y, Zhou B, Pache L, Chang M, Khodabakhshi AH, Tanaseichuk O, Benner C, and Chanda SK. 2019. Metascape provides a biologist-oriented resource for the analysis of systems-level datasets. Nat Commun 10:1523. 10.1038/s41467-019-09234-6

Peer) reviewing PDF | (2020:06:49732:2:0:NEW 9 Feb 2021) 


\section{Figure 1}

\section{The whole framework of the research process.}

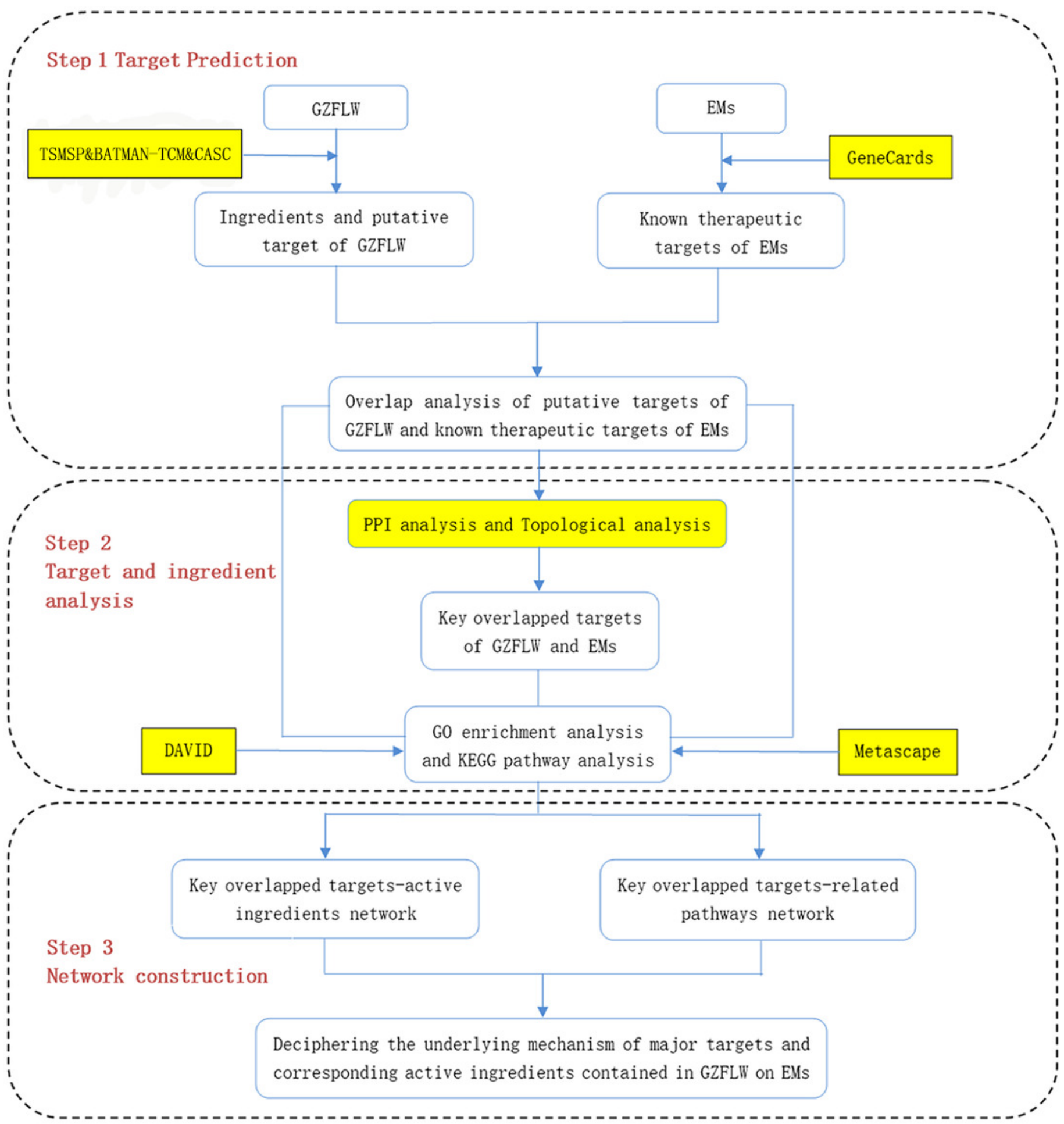


Figure 2

Herbs-Compounds Network.

The yellow nodes represent herbs in GZFLW, and the blue nodes represent active compounds. CT represents Cinnamon Twig, PC represents Paria cocos, CM represents Cortex Moutan, RPR represents Radix Paeoniae Rubra, and PK represents Peach kenel. The edges represent the relationship between them.

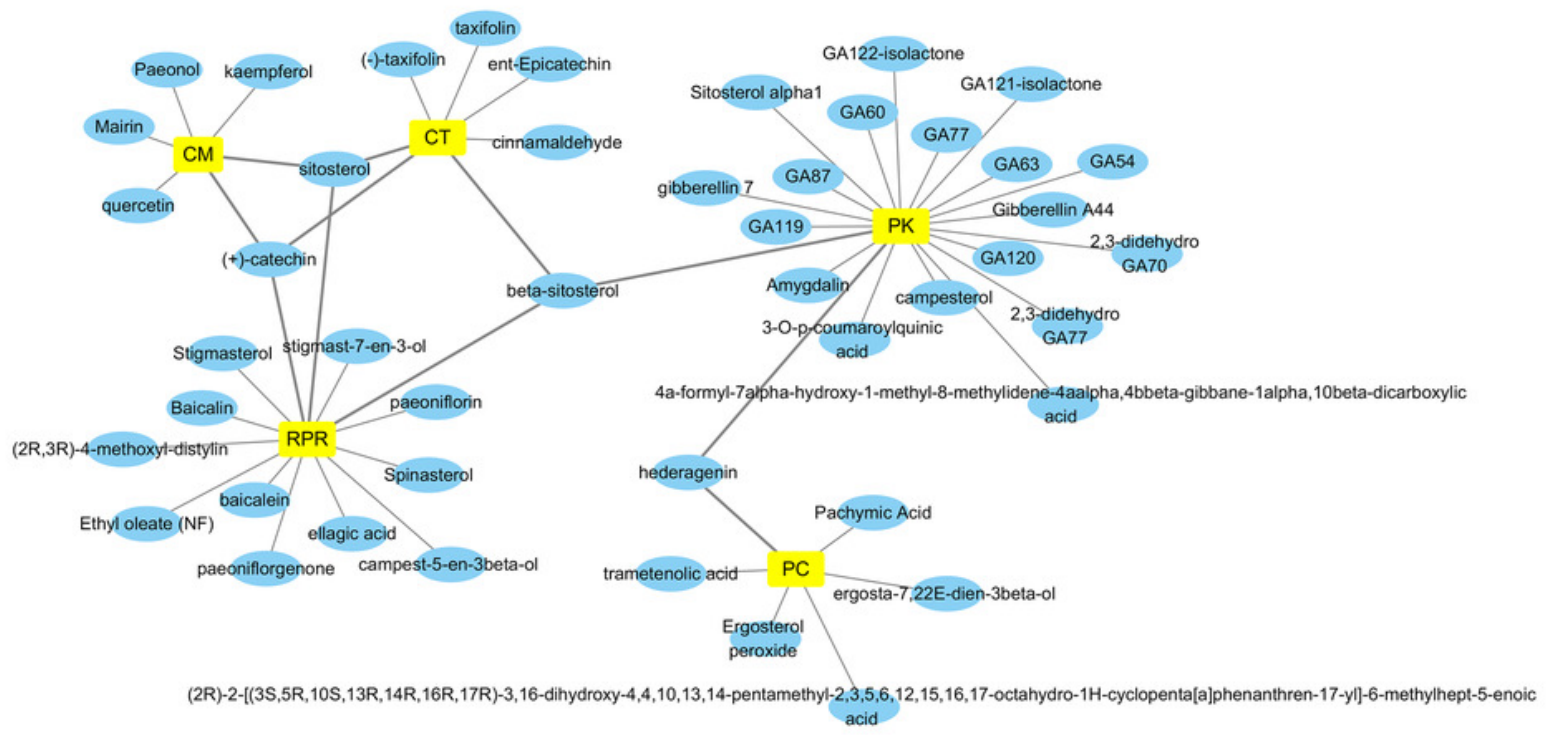


Figure 3

The Venn diagram of the targets both in endometriosis targets and GZFLW targets

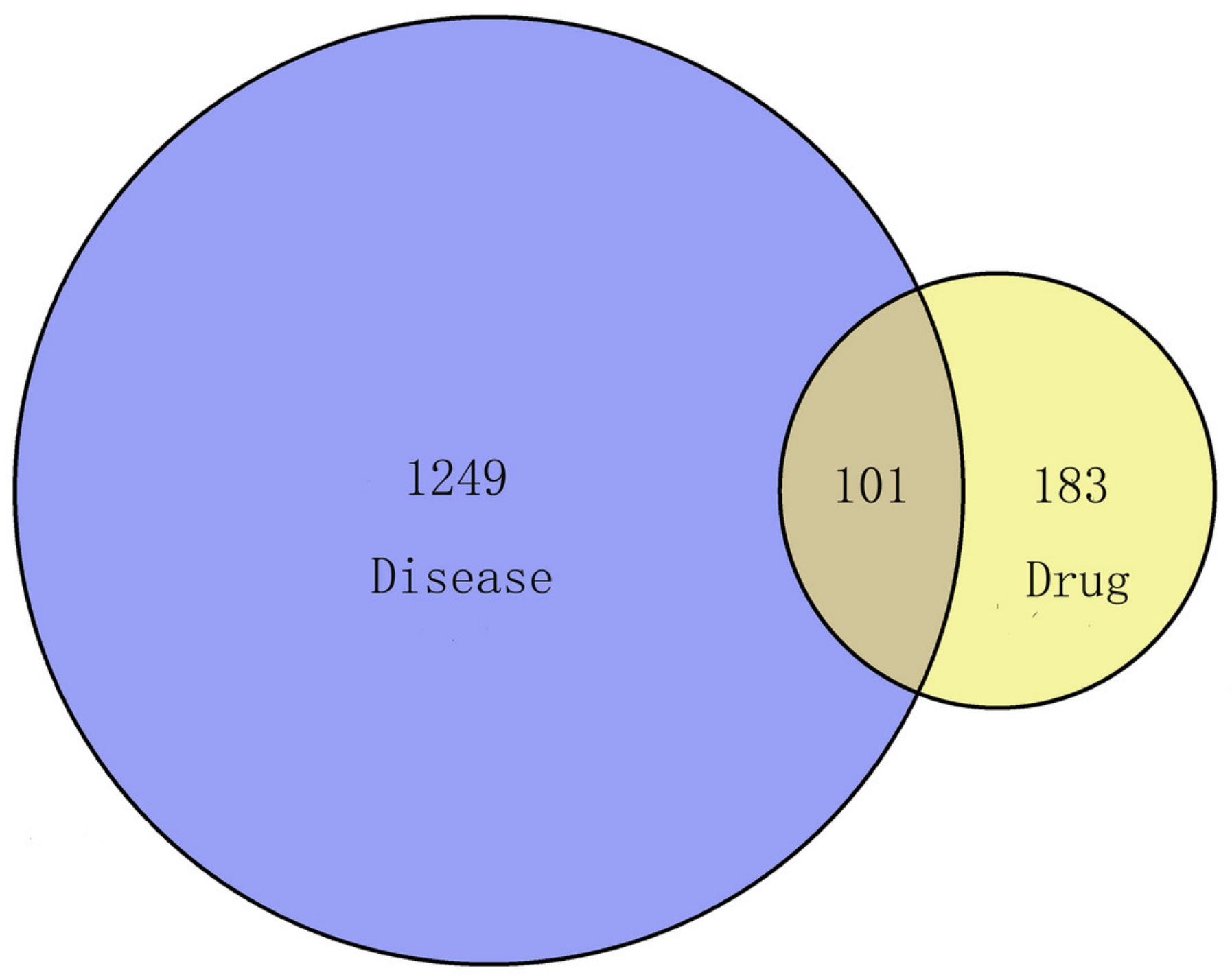




\section{Figure 4}

The network of 95 nodes.

The different color represents different nodes from each ingredients: The blue nodes represent the targets from $\mathrm{CM}$, the orange nodes represent the targets from $\mathrm{PC}$, the purple nodes represent the targets from RPR, and the yellow nodes represent the targets that are targeted by more than one ingredient. The node size is proportional to the target degree in the network. The edge color changes from light to dark reflect the betweenness value changes from low to high in the network. 


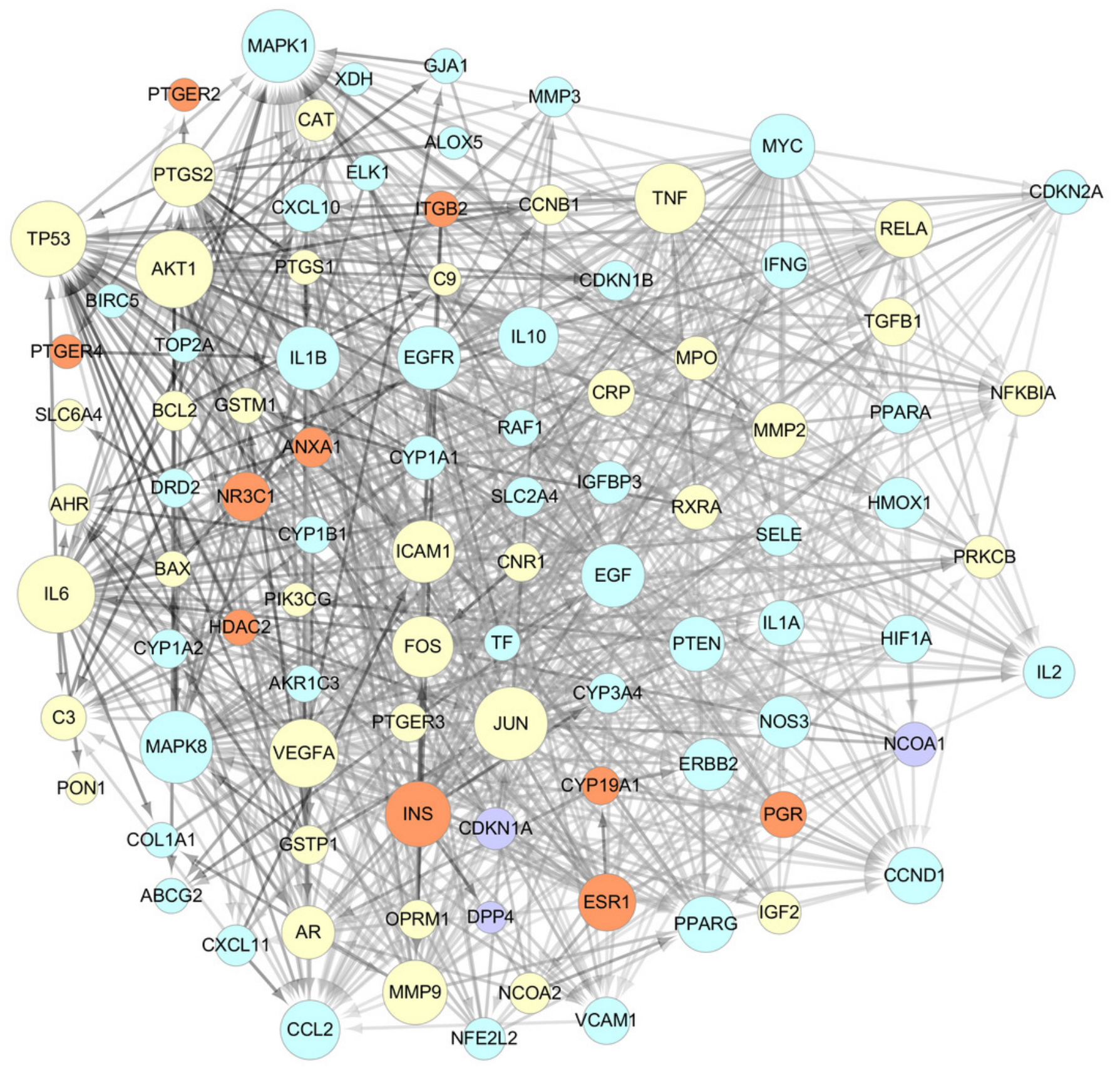




\section{Figure 5}

Hub nodes-Compunds Network.

The circle nodes represent the significant hub nodes, and the diamond nodes represent the compounds. The node size is proportional to the target degree in the network. 


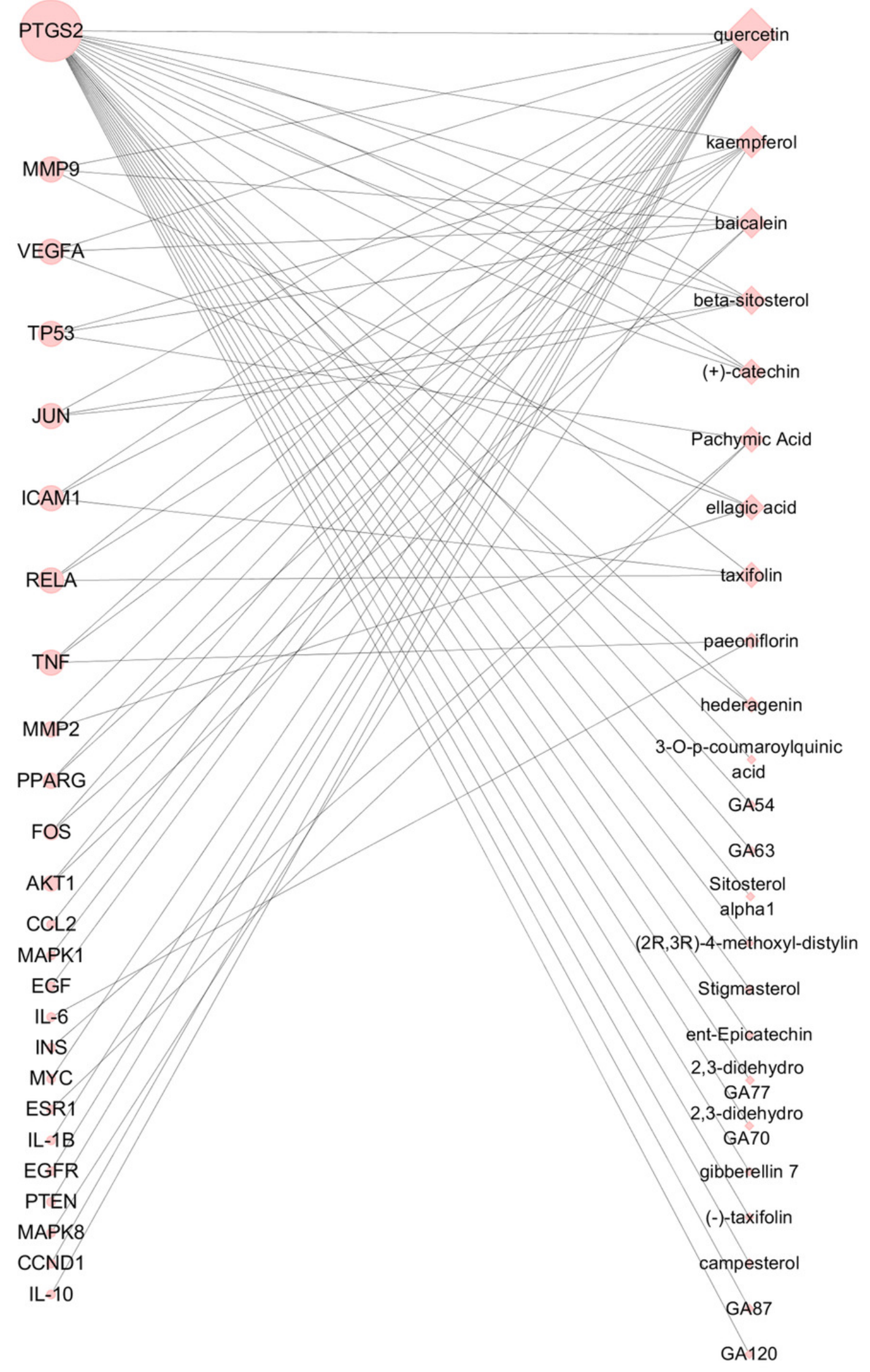


Figure 6

The molecular docking.

(A) TNF (PDBID: 2E7A) - campesterol molecular docking. (B) TNF (PDBID: 2E7A) - beta-

sitosterol molecular docking. (C) MMP9 (PDBID: 6ESM) - campesterol molecular docking. (D) MMP9 (PDBID: 6ESM) - beta-sitosterol molecular docking. The yellow sticks represent TNF and VEGFA. The blue sticks represent active compounds.
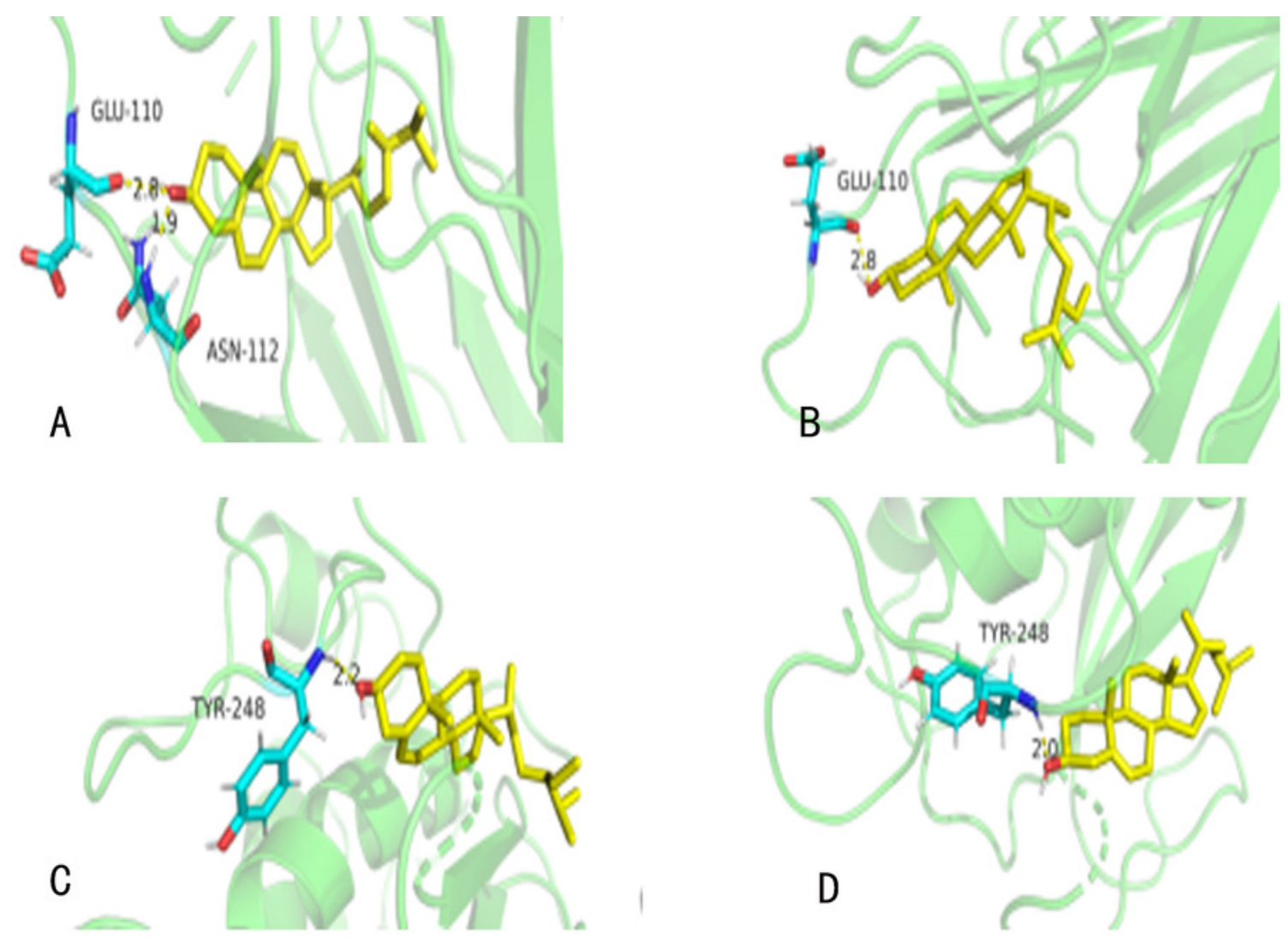
Figure 7

\section{The GO enrichment analysis of 101 nodes.}

\section{The orange part represents the Biological process. The blue part represents the Cellular component. The green part represents the Molecular function.}

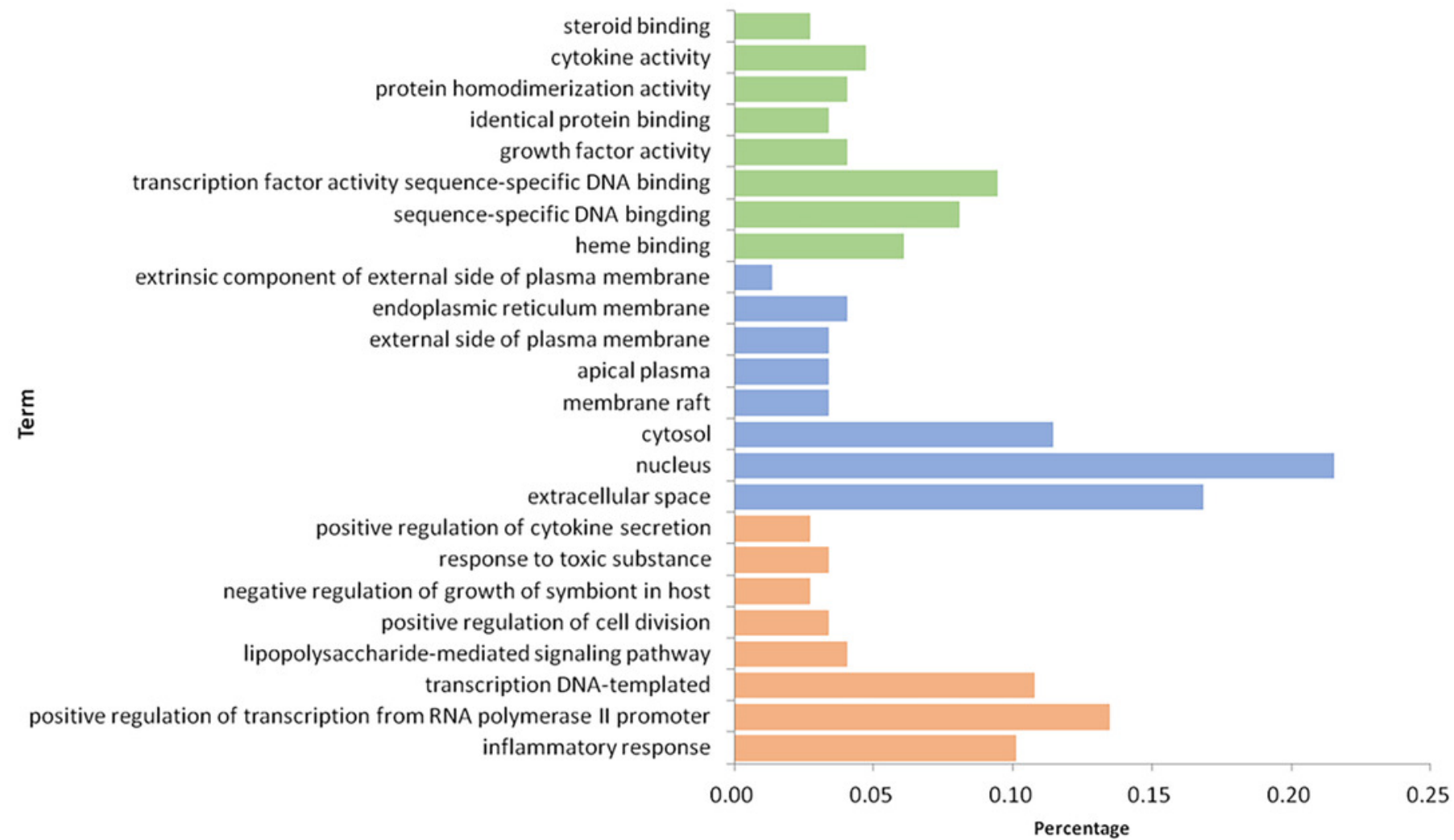




\section{Figure 8}

\section{Top 20 pathways of KEGG enrichment.}

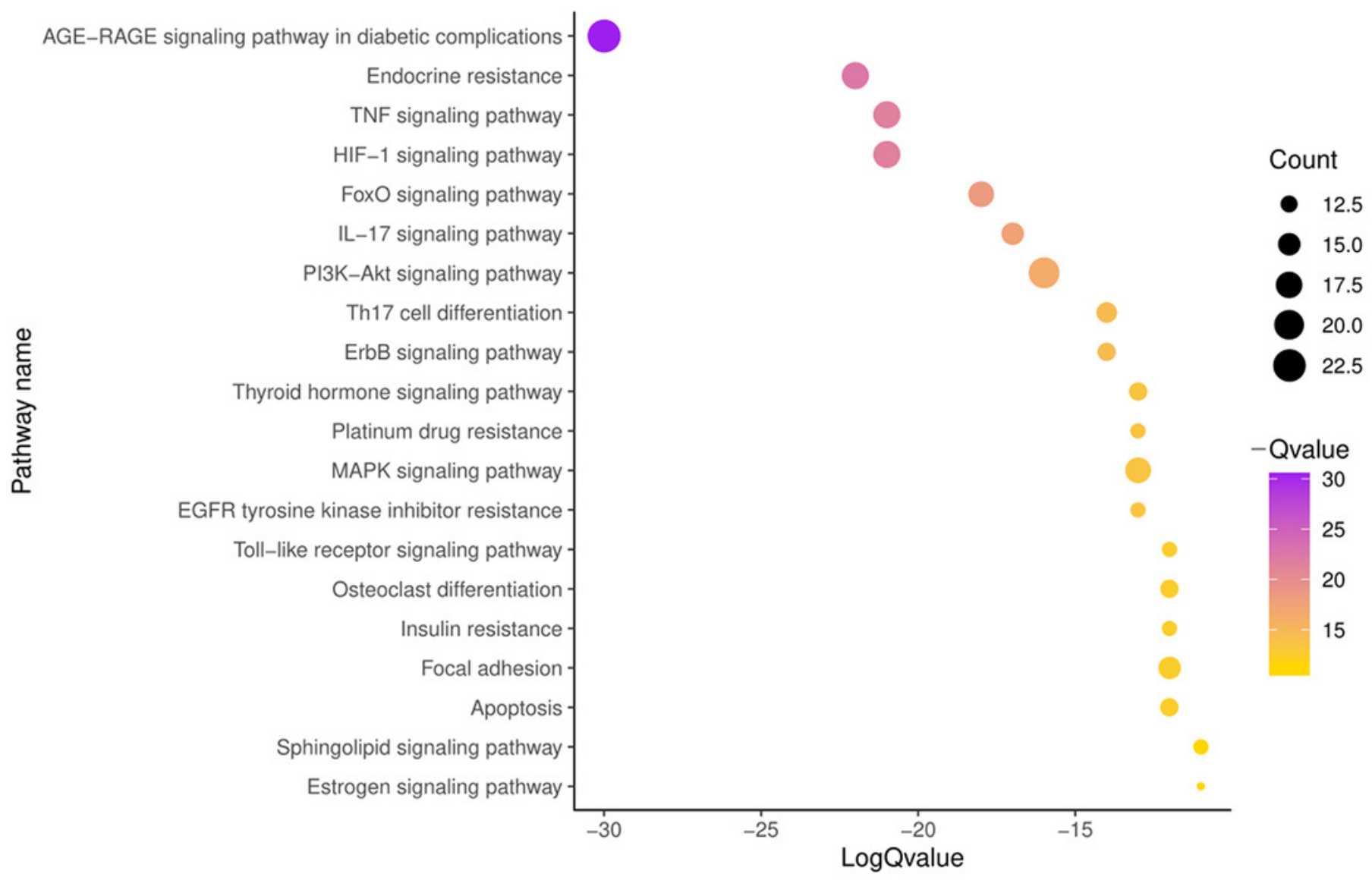


Figure 9

Target-Pathway Network.

The orange circle nodes represent the hub nodes, and the pink nodes represent the other nodes. The red diamond nodes represent the related pathways. The node size is proportional to the target degree in the network. 


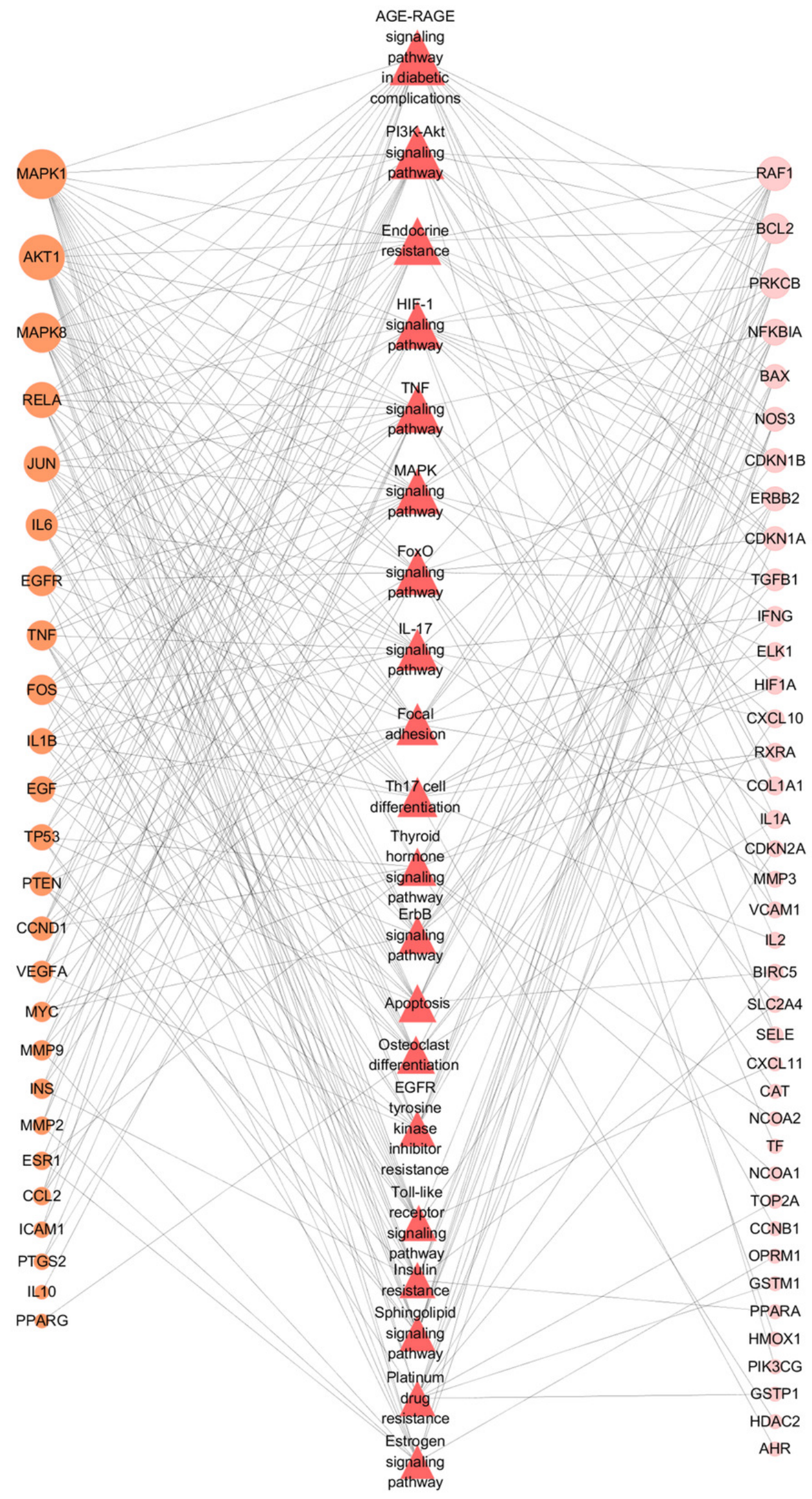




\section{Figure 10}

Primary endometrial stromal cells and vimentin staining

(A) Primary endometrial stromal cells. (B) The vimentin staining of cells.
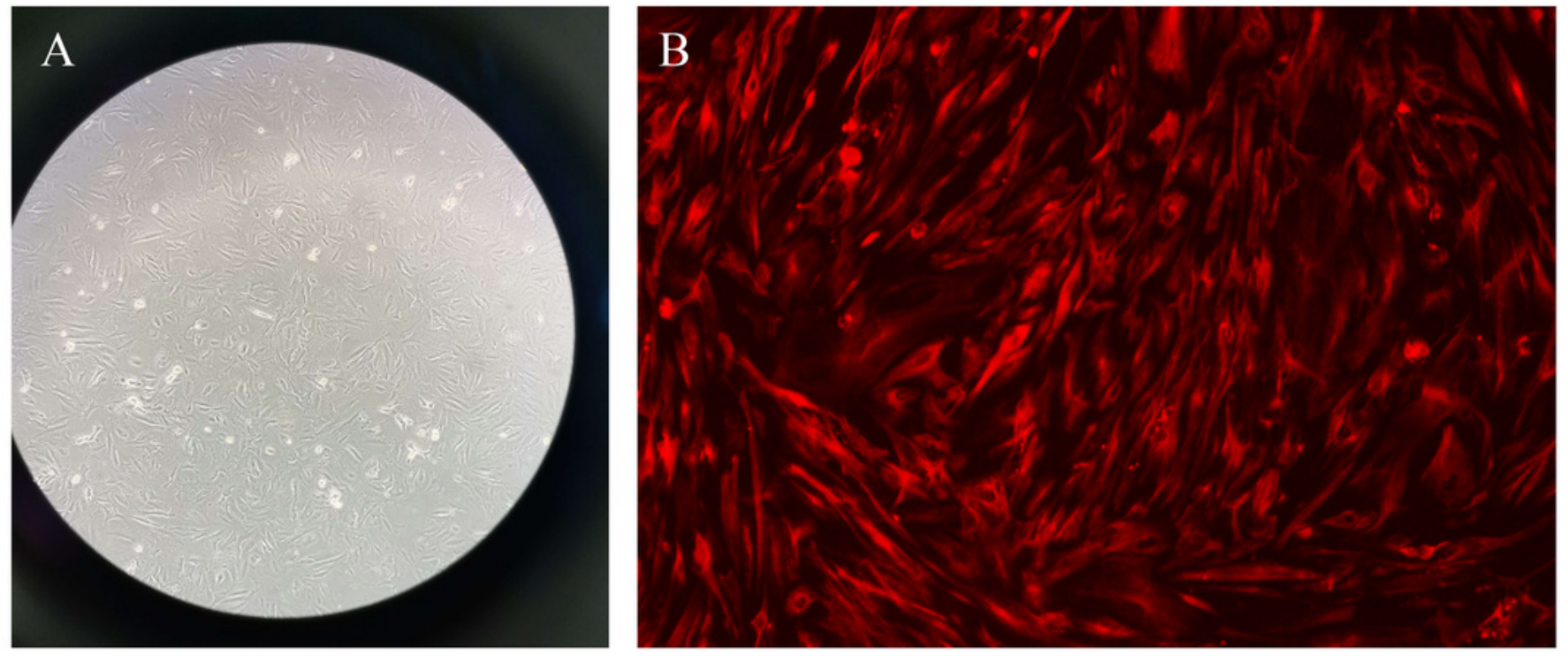
Figure 11

The results of MTT colorimetric assay

The cell proliferation of hEM15a cells was assessed using MTT assay. All results were shown as mean \pm standard deviation . $* p<0.05,{ }^{* *} p<0.01,{ }^{* * *} p<0.001$ versus designated group.
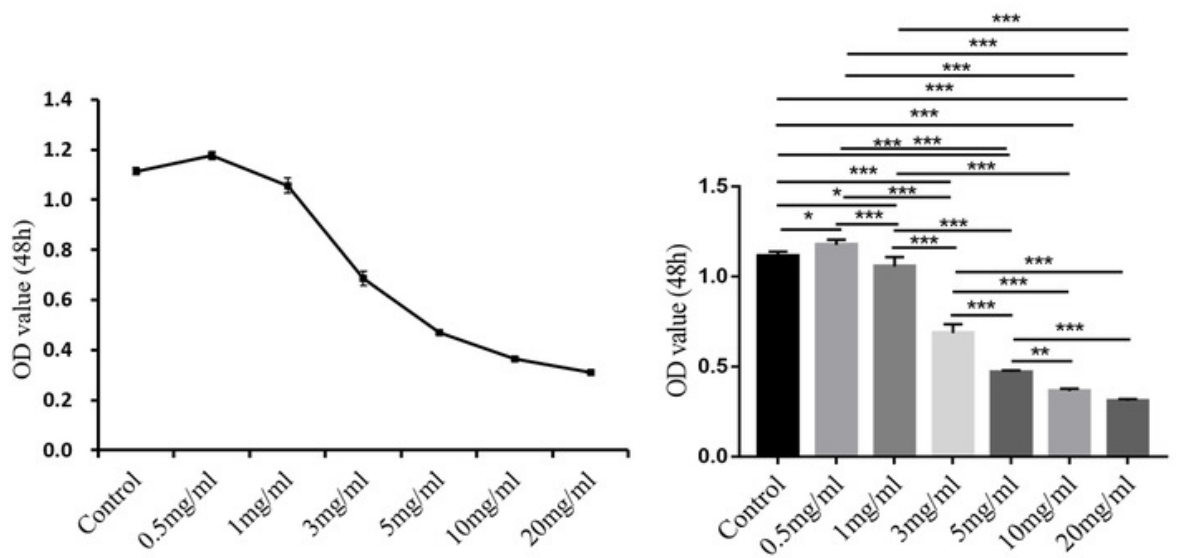


\section{Figure 12}

The results of Western blotting about different targets

The group 1-3 represented control group, and the group 4-6 represented GZFLW group. All results were shown as mean \pm standard deviation. ${ }^{* *} p<0.001$ versus designated group.

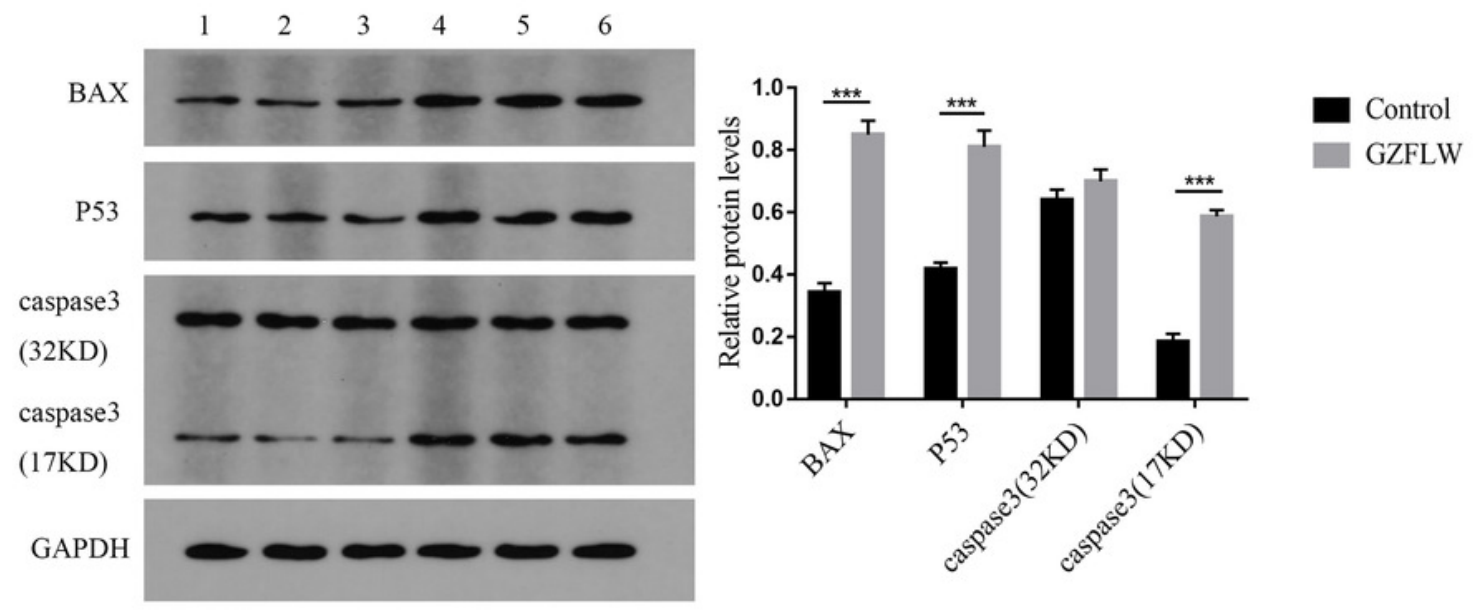


Figure 13

The apoptosis analysis of flow cytometry

The analysis of control group and GZFLW group was assessed by AnnexinV/PI staining using flow cytometry. The result was shown as mean \pm standard deviation. ${ }^{*} p<0.05$ versus designated group.
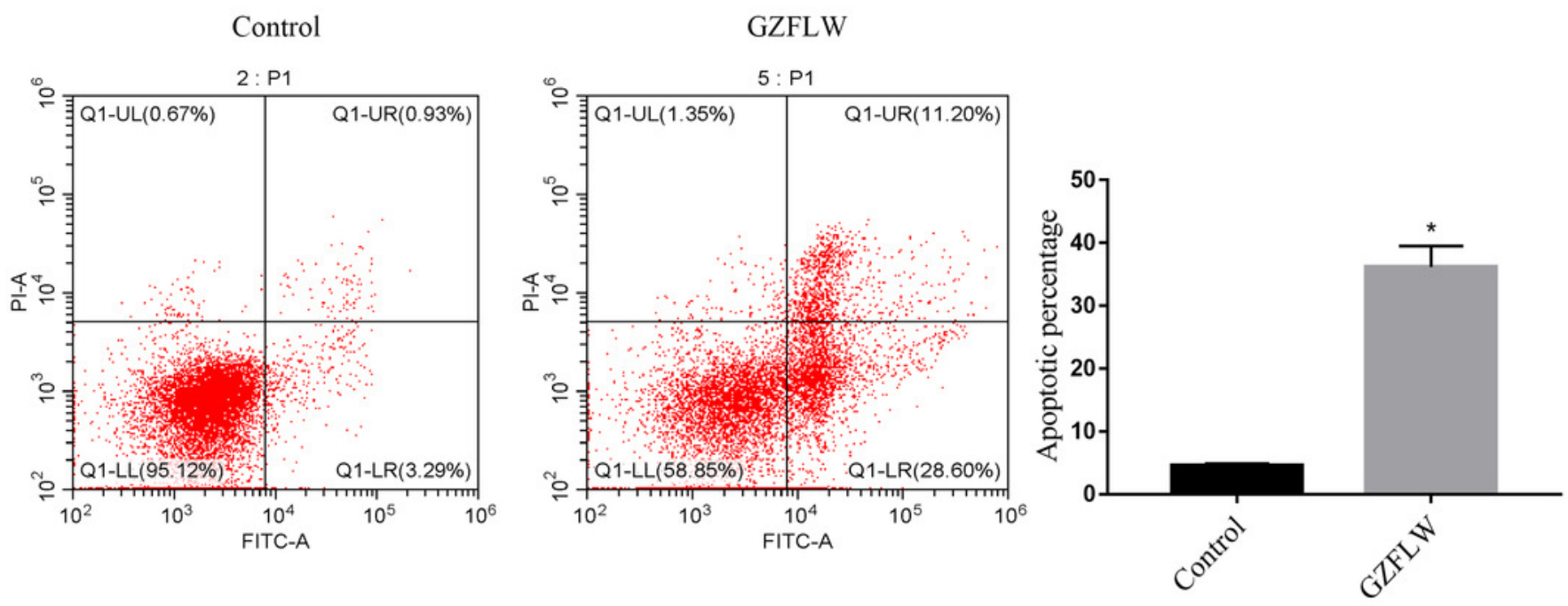
Table $\mathbf{1}$ (on next page)

Information for 89 active ingredients 


\begin{tabular}{|c|c|c|c|c|c|}
\hline herb name & Mol ID & compound & Code name & $\mathrm{OB} / \%$ & $\mathrm{DL} / \%$ \\
\hline \multirow[t]{7}{*}{ Cinnamon Twig } & MOL001736 & $(-)$-taxifolin & CT-1 & 60.51 & 0.27 \\
\hline & MOL004576 & taxifolin & CT-2 & 57.84 & 0.27 \\
\hline & MOL000492 & $(+)$-catechin & CT-3 & 54.83 & 0.24 \\
\hline & MOL000073 & ent-Epicatechin & CT-4 & 48.96 & 0.24 \\
\hline & MOL000358 & beta-sitosterol & CT-5 & 36.91 & 0.75 \\
\hline & MOL000359 & sitosterol & CT-6 & 36.91 & 0.75 \\
\hline & MOL000991 & cinnamaldehyde & CT-7 & 31.99 & 0.02 \\
\hline \multirow[t]{10}{*}{ Paria cocos } & MOL000282 & ergosta-7,22E-dien-3beta-ol & PC-1 & 43.51 & 0.72 \\
\hline & MOL000283 & Ergosterol peroxide & PC-2 & 40.36 & 0.81 \\
\hline & MOL000275 & trametenolic acid & PC-3 & 38.71 & 0.8 \\
\hline & MOL000296 & hederagenin & PC-4 & 36.91 & 0.75 \\
\hline & MOL000289 & Pachymic Acid & PC-5 & 33.63 & 0.81 \\
\hline & MOL000273 & (2R)-2-[(3S,5R,10S,13R,14R,16R,17R)- & PC-6 & 30.93 & 0.81 \\
\hline & & 3,16-dihydroxy-4,4,10,13,14-pentamethyl- & & & \\
\hline & & 2,3,5,6,12,15,16,17-octahydro-1H- & & & \\
\hline & & cyclopenta[a]phenanthren-17-yl]-6- & & & \\
\hline & & methylhept-5-enoic acid & & & \\
\hline \multirow[t]{6}{*}{ Cortex Moutan } & MOL000211 & Mairin & $\mathrm{CM}-1$ & 55.38 & 0.78 \\
\hline & MOL000492 & $(+)$-catechin & $\mathrm{CM}-2$ & 54.83 & 0.24 \\
\hline & MOL000098 & quercetin & $\mathrm{CM}-3$ & 46.43 & 0.28 \\
\hline & MOL000422 & kaempferol & CM-4 & 41.88 & 0.24 \\
\hline & MOL000359 & sitosterol & CM-5 & 36.91 & 0.75 \\
\hline & MOL000874 & Paeonol & CM-6 & 28.79 & 0.04 \\
\hline \multirow[t]{11}{*}{ Radix Paeoniae Rubra } & MOL001918 & paeoniflorgenone & RPR-1 & 87.59 & 0.37 \\
\hline & MOL006992 & (2R,3R)-4-methoxyl-distylin & RPR-2 & 59.98 & 0.3 \\
\hline & MOL000492 & $(+)$-catechin & RPR-3 & 54.83 & 0.24 \\
\hline & MOL001924 & paeoniflorin & RPR-4 & 53.87 & 0.79 \\
\hline & MOL000449 & Stigmasterol & RPR-5 & 43.83 & 0.76 \\
\hline & MOL001002 & ellagic acid & RPR-6 & 43.06 & 0.43 \\
\hline & MOL004355 & Spinasterol & RPR-7 & 42.98 & 0.76 \\
\hline & MOL002776 & Baicalin & RPR-8 & 40.12 & 0.75 \\
\hline & MOL005043 & campest-5-en-3beta-ol & RPR-9 & 37.58 & 0.71 \\
\hline & MOL006999 & stigmast-7-en-3-ol & RPR-10 & 37.42 & 0.75 \\
\hline & MOL000358 & beta-sitosterol & RPR-11 & 36.91 & 0.75 \\
\hline
\end{tabular}




\begin{tabular}{|c|c|c|c|c|c|}
\hline & MOL000359 & sitosterol & RPR-12 & 36.91 & 0.75 \\
\hline & MOL002714 & baicalein & RPR-13 & 33.52 & 0.21 \\
\hline & MOL002883 & Ethyl oleate (NF) & RPR-14 & 32.4 & 0.19 \\
\hline \multirow[t]{20}{*}{ Peach kenel } & MOL001351 & Gibberellin A44 & PK-1 & 101.61 & 0.54 \\
\hline & MOL001353 & GA60 & PK-2 & 93.17 & 0.53 \\
\hline & MOL001349 & $\begin{array}{l}\text { 4a-formyl-7alpha-hydroxy-1-methyl-8- } \\
\text { methylidene-4aalpha,4bbeta-gibbane- } \\
\text { 1alpha,10beta-dicarboxylic acid }\end{array}$ & PK-3 & 88.6 & 0.46 \\
\hline & MOL001344 & GA122-isolactone & PK-4 & 88.11 & 0.54 \\
\hline & MOL001329 & 2,3-didehydro GA77 & PK-5 & 88.08 & 0.53 \\
\hline & MOL001360 & GA77 & PK-6 & 87.89 & 0.53 \\
\hline & MOL001340 & GA120 & PK-7 & 84.85 & 0.45 \\
\hline & MOL001339 & GA119 & PK-8 & 76.36 & 0.49 \\
\hline & MOL001358 & gibberellin 7 & PK-9 & 73.8 & 0.5 \\
\hline & MOL001342 & GA121-isolactone & PK-10 & 72.7 & 0.54 \\
\hline & MOL001361 & GA87 & PK-11 & 68.85 & 0.57 \\
\hline & MOL001355 & GA63 & PK-12 & 65.54 & 0.54 \\
\hline & MOL001352 & GA54 & PK-13 & 64.21 & 0.53 \\
\hline & MOL001328 & 2,3-didehydro GA70 & PK-14 & 63.29 & 0.5 \\
\hline & MOL001323 & Sitosterol alpha1 & PK-15 & 43.28 & 0.78 \\
\hline & MOL001368 & 3-O-p-coumaroylquinic acid & PK-16 & 37.63 & 0.29 \\
\hline & MOL000493 & campesterol & PK-17 & 37.58 & 0.71 \\
\hline & MOL000296 & hederagenin & PK-18 & 36.91 & 0.75 \\
\hline & MOL000358 & beta-sitosterol & PK-19 & 36.91 & 0.75 \\
\hline & MOL001320 & Amygdalin & PK-20 & 4.42 & 0.61 \\
\hline
\end{tabular}


Table 2 (on next page)

Information for 25 hub targets 


\begin{tabular}{lccccccccccc}
\hline NO. & gene & Degree & BC & NO. & gene & Degree & BC & NO. & gene & Degree & BC \\
\hline 1 & IL6 & 23 & 9.1459 & 10 & AKT1 & 20 & 6.5181 & 19 & MYC & 17 & 3.400 \\
2 & JUN & 23 & 8.9097 & 11 & INS & 20 & 5.9093 & 20 & IL10 & 16 & 2.466 \\
3 & TNF & 23 & 8.8208 & 12 & FOS & 19 & 6.1117 & 21 & PTEN & 16 & 3.013 \\
4 & MAPK1 & 22 & 8.6040 & 13 & ICAM1 & 18 & 3.4111 & 22 & ESR1 & 15 & 1.535 \\
5 & TP53 & 22 & 7.9533 & 14 & PTGS2 & 18 & 5.1667 & 23 & PPARG & 15 & 2.207 \\
6 & EGF & 21 & 6.3421 & 15 & CCL2 & 17 & 2.6724 & 24 & RELA & 15 & 2.605 \\
7 & MAPK8 & 21 & 7.5937 & 16 & CCND1 & 17 & 3.3755 & 25 & MMP2 & 14 & 2.048 \\
8 & MMP9 & 21 & 6.9767 & 17 & EGFR & 17 & 3.4135 & & & & \\
9 & VEGFA & 21 & 8.0120 & 18 & IL1B & 17 & 3.2828 & & & &
\end{tabular}




\section{Table 3 (on next page)}

The docking information of 13 targets with the corresponding compounds

The values in this table are docking scores that between targets ans compounds. 
Pachymic Acid taxifolin quercetin paeoniflorin beta-sitosterol campesterol hederagenin

\begin{tabular}{|c|c|c|c|c|c|c|c|}
\hline AKT1-4EKL & -5.02 & -7.65 & -5.58 & -4.19 & -8.56 & -8.27 & -7.61 \\
\hline AKT1-6S9W & -7.85 & -6.88 & -5.08 & -4.21 & -8.59 & -9.99 & -8.79 \\
\hline MAPK1-6RQ4 & -7.25 & -6.22 & -5.26 & -3.61 & -6.25 & -7.16 & -8.16 \\
\hline MAPK1-1WZY & -6.62 & -6.51 & -4.3 & -3.7 & -8.09 & -6.64 & -8.02 \\
\hline TNF-2E7A & -8.68 & -6.61 & -7.22 & -4.2 & -9.17 & -10.31 & -10.69 \\
\hline TNF-2ZJC & -4.25 & -5.06 & -3.45 & -2.55 & -4.9 & -5.44 & -4.98 \\
\hline TNF-2AZ5 & -6.07 & -4.57 & -4 & -3.55 & -6.08 & -7.16 & -6.79 \\
\hline TP53-4MZI & -7.28 & -7.11 & -5.71 & -5.12 & -7.65 & -6.27 & -7.84 \\
\hline TP53-6FF9 & -5.44 & -4.19 & -4.07 & -2.93 & -5.62 & -5.14 & -6.28 \\
\hline VEGFA-4WPB & -6.7 & -5.81 & -5.36 & -4.09 & -7.48 & -7.01 & -7 \\
\hline VEGFA-3QTK & -4.31 & -3.99 & -3.15 & -2.58 & -4.47 & -6.18 & -5.61 \\
\hline VEGFA-4QAF & -4.76 & -5.41 & -3.96 & -2.4 & -5.93 & -5.64 & -5.58 \\
\hline MMP9-6ESM & -7.83 & -9.32 & -7.55 & -6.24 & -9.03 & -9.36 & -8.83 \\
\hline MMP9-4WZV & -6.09 & -7.53 & -7.53 & -4.45 & -10.01 & -10.5 & -6.84 \\
\hline JUN-5T01 & -5.46 & -4.15 & -4.63 & -1.96 & -5.51 & -4.85 & -7.6 \\
\hline JUN-1T2K & -6.28 & -3.07 & -3.3 & -1.81 & -5.17 & -5.03 & -6.43 \\
\hline MAPK8-3VUK & -6.25 & -5.87 & -6.17 & -4.31 & -7.05 & -8.9 & -6.99 \\
\hline MAPK8-4L7F & -4.96 & -5.94 & -4 & -3.12 & -5.5 & -5.34 & -6.96 \\
\hline INS-4AJX & -8.6 & -6.48 & -6.66 & -6.32 & -8.72 & -9.18 & -7.92 \\
\hline INS-4CY7 & -6.62 & -4.84 & -5.6 & -4.13 & -6.56 & -6.08 & -7.58 \\
\hline EGF-IJL9 & -7.42 & -7.12 & -7.29 & -3.89 & -7.94 & -7.58 & -9.14 \\
\hline IL6-4O9H & -6.49 & -5.34 & -4.66 & -3.81 & -5.96 & -5.95 & -6.48 \\
\hline IL6-1ALU & -5.23 & -3.94 & -3.74 & -2.5 & -4.58 & -5.09 & -6.45 \\
\hline
\end{tabular}


